\title{
A compact and robust method for full Stokes spectropolarimetry
}

\author{
William Sparks, ${ }^{1, *}$ Thomas A. Germer, ${ }^{2}$ John MacKenty ${ }^{1}$ and Frans Snik ${ }^{3}$ \\ ${ }^{1}$ Space Telescope Science Institute, 3700 San Martin Drive, Baltimore, MD 21218, USA \\ ${ }^{2}$ National Institute of Standards and Technology, 100 Bureau Drive, Gaithersburg, MD \\ 20899, USA \\ ${ }^{3}$ Sterrewacht Leiden, Universiteit Leiden, Oortgebouw 570, Niels Bohrweg 2, 2333 CA \\ Leiden, The Netherlands \\ *Corresponding author: sparks@stsci.edu
}

\begin{abstract}
We present an approach to spectropolarimetry which requires neither moving parts nor time dependent modulation, and which offers the prospect of achieving high sensitivity. The technique applies equally well, in principle, in the optical, UV or IR. The concept, which is one of those generically known as channeled polarimetry, is to encode the polarization information at each wavelength along the spatial dimension of a 2D data array using static, robust optical components. A single two-dimensional data frame contains the full polarization information and can be configured to measure either two or all of the Stokes polarization parameters. By acquiring full polarimetric information in a single observation, we simplify polarimetry of transient sources and in situations where the instrument and target are in relative motion. The robustness and simplicity of the approach, coupled to its potential for high sensitivity, and applicability over a wide wavelength range, is likely to prove useful for applications in challenging environments such as space. (c) 2018 Optical Society of America
\end{abstract}

OCIS codes: $120.5410,260.5430$.

\section{Introduction}

The polarization of light provides a versatile suite of remote sensing diagnostics. In astronomy, polarization is used to study the Sun and Solar System, stars, dust, supernova remnants, 
and high-energy extragalactic astrophysics [1]. The astrophysical mechanisms by which polarized light is produced range from scattering phenomena to the interaction between high energy charged particles, and magnetized plasmas. Beyond astronomy, polarization is used in remote sensing, medical diagnostics, defense, biophysics, microscopy, and fundamental experimental physics, e.g. [2].

Accurate, precision polarimetric methods usually require rapidly modulating, often fragile, parts and are inherently monochromatic, e.g. photoelastic modulators (PEMs), ferroelectric liquid crystals or liquid crystal variable retarders (LCVRs) in tandem with phase locked photomultipliers, or synchronized charge shuffling on a charge-coupled device (CCD) detector for area detection [1]. Lower accuracy techniques typically require sequential measurements of the target using rotating waveplates and polarization analyzers. Here we describe a method to encode polarimetric information over a wide spectrum in a single data frame, using static optics. This approach alleviates errors introduced by the need to match sequentially acquired data, and eliminates the need for fragile or rapid modulation, yet may be able to accomplish high accuracy, precision measurements. The methods, of course, have their own implicit sensitivities and concerns, as we discuss below.

A particular interest of the authors, which serves as a useful illustrative example, is the use of precision circular polarization spectroscopy as a remote sensing biosignature and a potentially valuable tool in searches for biological processes elsewhere in the Universe. The circular polarization spectrum is sensitive to the presence of molecular homochirality, a strong biosignature, through the combined optical activity and homochirality of biological molecules $[3,4]$. Biologically-induced degrees of circular polarization have been found in the range $10^{-2}$ to $10^{-4}$ for a variety of photosynthetic samples, with an important correlation between the intensity spectrum and polarization spectrum [3]. Hence, precision full Stokes polarimetry and wide spectral coverage are required. Furthermore, the target scene and instrumentation may be in rapid relative motion, compounding the difficulties of acquiring the data using traditional polarimetric techniques. A large number of photons must be accumulated in a short period of time. The techniques presented in this paper may provide a means to make this type of polarization measurement, in addition to providing a robust method for acquiring less precise spectropolarimetry in a straightforward fashion. Furthermore, the approach is applicable across a wide wavelength range, and, as well as in the visible, can work equally well in the ultraviolet, where for example chiral electronic signatures are generally strongest, to the infrared, where polarimetry goes hand in hand with probes into the geometry and physical characteristics of dusty regions of the universe.

A variety of similar concepts are available under the generic title of "channeled polarimetry" [2]. These typically fall into two classes: channeled imaging polarimetry (CIP) and channeled spectropolarimetry (CS), following terminology of [2]. To simplify, the CS meth- 
ods typically encode the polarization information as an amplitude modulation directly on the spectrum, derived from a polarization optic whose retardance is a function of wavelength. As an example, the spectral modulation principle for linear spectropolarimetry [5] can reach a precision of at least $2 \times 10^{-4}$ [6]. The CIP methods, by contrast, use a polarization optic whose retardance is spatially varying, so that the polarization information is encoded as a set of spatial fringes onto an image [7]. These two approaches, as well as a number of technical issues that arise in each case, are described in some detail in [2]. Previous authors have used multiorder retarders, birefringent wedges, pairs of birefringent wedges, and Savart plates individually or in combination for these two applications [5,7-16]. Typically, the polarization information is extracted from the data using Fourier methods. Another approach to single-shot imaging polarimetry and spectropolarimetry is the wedged double Wollaston device, which yields multiple images on a detector with polarization axes at different angles and allows retrieval of the Stokes parameters through combinations of the images [17-19].

The approach explored in the current paper is to disperse the spectral and polarimetric information along two orthogonal directions, a "spectral" dimension for the spectroscopy and a "spatial" dimension for the polarimetry. The amplitude modulation of the encoding of the polarization information is independent of the choice of spectral resolution. The two aspects of the measurement, the spectroscopy and the polarimetry, may be optimized independently. The complete spectropolarimetric information is encoded on a single data frame, and may be derived using straightforward analytical techniques.

Poisson photon counting statistics play a critical role in astronomical polarimetry. To measure a polarization degree of $10^{-n}$, it is necessary to collect (at least) $10^{2 n}$ photons. For example, to measure $p \approx 10^{-4}$, it is necessary to accumulate $10^{8}$ photons. A typical astronomical CCD has a well-depth $\sim 10^{5}$ electrons per pixel, requiring $10^{3}$ pixel readouts. If the data are needed in, say, $1 \mathrm{~s}$ in one pixel, this multi-readout approach becomes prohibitive. A solution is to spread the illumination across many pixels, as is done for high signal-tonoise-ratio photometry with the Hubble Space Telescope [20]. Making a virtue of necessity, if we use optics which spread the light of a spectrum perpendicular to the spectrum, then we can exploit the width of the broadened spectrum to encode the polarimetric data.

Sections 2 and 4 discuss a variety of configurations that accomplish this goal. Sec. 2 starts with linear polarization (equivalently any two of the Stokes polarization parameters), followed by a discussion of our analysis methods in Sec. 3. Configurations that enable full Stokes spectropolarimetry are presented in Sec. 4. Sec. 5 describes practical implementation, sensitivities, and an approach based on calibration. Sec. 6 provides an example application. Finally, we make some conclusions in Sec. 7.

The different embodiments of the underlying approach described in Secs. 2 and 4 highlight different aspects of the method. In the end, we anticipate that the most useful realizations of 
the concept will be the double wedge for linear polarimetry, Sec. 2B, and the double-double wedge for full Stokes spectropolarimetry, Sec. 4.B.3. The other subsections introduce new ideas incrementally, while these two sections capture the final products for the two types of polarimetry.

We use the conventional Stokes vector formalism to quantify the polarization of light with $S \equiv(I, Q, U, V)$ where $I$ is the total intensity; $Q, U$ decribe the linear polarization and $V$ the circular polarization. The normalized Stokes parameters $(q, u, v)=(Q, U, V) / I$ represent

the fractional polarization state. The degree of polarization is given by $p=\sqrt{q^{2}+u^{2}+v^{2}}$ and the direction of linear polarization by $\psi=\frac{1}{2} \tan ^{-1}(u / q)$.

\section{Concept for Linear Polarimetry}

We envisage a spectrum of light broadened in a direction orthogonal to the dispersion direction and sensed using a two dimensional area array such as a CCD. This broadening can be spread along a segment of a conventional long slit spectrograph, for example, with the length of the entrance slit providing the spatial dimension in the detected two dimensional spectrum. To introduce amplitude modulation along the slit ( $x$ direction), we introduce a retardance gradient $\phi(x)$ along $x$ using a birefringent wedge (or wedges) followed by a polarization analyzer, such as a dichroic polarizer or polarizing prism (see Figs. 1 and 2). It would be possible to carry out the polarization analysis immediately in front of the detector array, as in [7]. However, performing the polarization analysis as early as possible in the optical path yields better robustness against polarization introduced by the instrumentation optics. Furthermore, the polarization optics can be more compact and easier to characterize, since light from all wavelengths is analyzed using the same optical elements. Hence, we prefer to insert the polarization optics immediately adjacent to the spectrograph's entrance slit, and allow a long-slit spatial segment to project through the instrument to become the detector array's spatial dimension.

\section{A. Single birefringent wedge}

To lay the groundwork, we initially consider just a single birefringent wedge. The wedge thickness gradient is oriented along the slit, while its fast axis is oriented $45^{\circ}$ with respect to the slit. The analyzer's transmission axis is parallel to the slit, though it could alternatively be orthogonal to it. If we define the Stokes $Q$ direction as also being parallel to the slit, then we can consider a uniformly illuminated slit with the beam entering the slit, orthogonal to the slit plane. If the incoming light is polarized with its electric field along the slit $(q=1)$, at the hypothetical tip of the wedge where the retardance is zero, the polarized light passes through the retarder and analyzer without hinderance. Moving along the slit, the retardance increases to the point where it becomes quarter-wave, and the light is converted to circularly polarized 
light after the retarder, and half of the light transmits through the analyzer. As $x$, and the retardance, increase together, it reaches the point where there is half wave retardance. At that point the polarization is rotated $90^{\circ}$ after the retarder, and none of the light transmits through the analyzer. At the same distance further along the slit, the retardance is fullwave, the light is completely transmitted, and the cycle is complete. Note that for typical birefringent materials, the spatial distance $x$ corresponding to one wavelength of retardance will depend on the wavelength. In the absence of dispersion, the spatial modulation frequency is $\propto 1 / \lambda$.

Circularly polarized light $(v=1)$ is half transmitted at zero retardance (circularly polarized light passing through the analyzer). When the retardance reaches quarter-wave, the light becomes linearly polarized along the slit direction and all of the light passes the analyzer. When the retardance is half-wave, the sign of the circular polarization is flipped $(v=-1)$, and again, half of the light is transmitted through the analyzer. Hence, the modulation for $v=1$ is similar to the modulation due to $q=1$, but out of phase by a distance corresponding to one quarter wave of retardance. Light polarized linearly at $45^{\circ}(u=1)$, i.e., along the retarder fast axis, is unaffected by the variable retardance along the slit. However, if we precede the birefringent wedge by an achromatic (or superachromatic) quarter wave retarder, with fast axis along the slit, then the circular polarization parameter $V$ is interchanged with $U[5]$. Now $V$ is unaffected by the variable retardance and causes no spatial modulation, while $U$ causes spatial amplitude modulation, a quarter wave out of phase from $Q$. If the input Stokes vector is $(I, Q, U, V)$, the output intensity $I_{\|}$in the spatial direction is

$$
I_{\|}=0.5(I+Q \cos \phi+U \sin \phi)
$$

The retardance $\phi$ maps onto the distance $x$ along the slit according to $\phi=2 \pi(x / X)$, where the distance corresponding to a single wave of retardance change is $\left.X=\lambda /\left(\left|n_{o}-n_{e}\right| \tan \xi\right)\right\}, \xi$ is the wedge angle, and $n_{o}$ and $n_{e}$ are the refractive indices for the $o$ and $e$-beams, respectively. If the circular polarization $V$ is desired rather than $U$, then the quarter wave retarder can be omitted.

If a beam splitting polarizing prism (e.g. a Wollaston prism) is used as the polarization analyzer, then two versions of the spectra are obtained on the detector. The intensity $I_{\perp}$ of the orthogonally polarized beam is

$$
I_{\perp}=0.5(I-Q \cos \phi-U \sin \phi) .
$$

The difference of Eqs. (1) and (2), divided by their sum, assuming any transmission differences have been removed, gives

$$
I^{\prime}=\left(I_{\|}-I_{\perp}\right) /\left(I_{\|}+I_{\perp}\right)=q \cos \phi+u \sin \phi
$$


Table 1 summarizes this and other algebraic expressions for the spatial modulation in subsequent configurations discussed below. An alternative way to express Eqs. (1)-(3) is

$$
\begin{aligned}
I_{\|} & =0.5 I(1+p \cos (\phi-2 \psi)) \\
I_{\perp} & =0.5 I(1-p \cos (\phi-2 \psi)) \\
I^{\prime} & =p \cos (\phi-2 \psi),
\end{aligned}
$$

where the position angle $\psi$ of linear polarization is given by $\psi=\frac{1}{2} \tan ^{-1}(U / Q)$. From Eq. (4) it is apparent that the spatially modulated profile has an amplitude of modulation equal to the degree of polarization, and a (spatial) phase zero point that reveals the angle of polarization.

It is important in using Eqs. (1) and (2) that there not be any signficant intensity variations along the slit on length scales of order $X$. However, in the "dual beam" version, Eq. (3), the total intensity $I$ along the slit has been eliminated [5]. This potentially offers a means to retain some spatial resolution along the slit. For example, the image of a star can have very large intensity changes along a spectrograph slit, though its polarization is unchanged. Provided the extent of the image is sufficient to encode the sine and cosine terms in Eq. (3), we may derive its polarization even in the presence of quite strong intensity changes. In Eqs. (1) and (2), intrinsic intensity changes would be mixed with amplitude modulation produced by polarization. Hence, care needs to be taken in matching the spatial extent of the instrumental point spread function to the projected scale of the retardance variation. Use of Eq. (3) is more robust against this constraint.

In practice, wedge components available off the shelf are relatively thick. This introduces a multiorder retarder effect, exploited in the SPEX concept [5]. A thick birefringent material, followed by analysis optics, such as those employed here, for a single location on the slit, yields spectral modulation, used to measure the polarization in [5]. Hence, using a single wedge, the resulting fringes from polarized light have a relatively pronounced "slope" because the retardance, implicitly, is varying as a function of both wavelength and spatial direction. From above, the complete expression for $\phi$ is $\phi=2 \pi x\left(n_{o}-n_{e}\right) \tan \xi / \lambda$. Therefore a constant phase $\phi$, which defines the appearance of the fringes on the detector, occurs for $x=\left[\phi /\left(2 \pi\left(n_{e}-n_{o}\right) \tan \xi\right)\right] \lambda$. The thickness of the wedge on the narrow edge effectively adds a constant to $\phi$ and hence increases the slope of $x$ vs. $\lambda$.

In principle, analysis methods applied to the orthogonal dimension, discussed in Sec. 4, can remove this spectral modulation. The slope also translates to a constraint on the spectral resolution, as it must be sufficient to separate the fringes. 


\section{B. Double wedge}

An alternative strategy to alleviate the spectral resolution constraint, and at the same time simplify and render the analysis more robust, is to compound the first wedge with an identical second wedge reversed in direction, with its fast axis orthogonal to the fast axis of the first wedge. The resulting optic, also used in Babinet-Soleil compensators, is convenient to work with (it is now a rectangular cuboid, see Fig. 3), and the reversed signs of birefringence conspire to yield a zero retardance at the point where the two retardances are equal, expected to be close to the center of the optic. This is the configuration discussed by $[2,7]$ in the context of imaging polarimetry. In imaging polarimetry, spectral modulation across the bandpass would be highly problematic, and this configuration goes some way towards eliminating it. [2] discusses other ways to mitigate birefringence dispersion. When applied to spectropolarimetry, as here, the effect is to remove the slope from the fringes and cause them to be approximately parallel in the spectral dimension, with spacing convergence to the blue, as a given amount of retardance corresponds to a greater number of wavelengths.

Using Mueller matrix algebra, it can be shown that the amplitude modulated profiles for this configuration are, for the single beam, with a quarter wave plate used, as above, to impose a sensitivity to Stokes $U$ rather than Stokes $V$ :

$$
I_{\|}=0.5(I+Q \cos 2 \phi+U \sin 2 \phi)
$$

and for the dual beam

$$
I^{\prime}=q \cos 2 \phi+u \sin 2 \phi
$$

The essential characteristics of the compound wedge profiles are the same as the single wedge, though the spatial frequency is doubled and the multiorder retarder effect with wavelength is removed making the fringes essentially parallel to the dispersion direction. The double wedge also yields a quasi-zero order retarder, which has much smaller temperature dependence than a single wedge.

Fig. 4 shows simulations of data frames obtained with $100 \%$ polarized light for each of the configurations discussed here, for $Q, U$ and $V$ polarized light separately. To illustrate the concepts of this section, Fig. 5 shows actual long-slit spectra obtained using one and two quartz birefringent wedges with a $3^{\circ}$ wedge angle, installed in a simple slit spectrograph. We inserted the quartz wedges immediately after the entrance slit of the spectrograph, together with a quarter wave plate and analyzer as discussed. Fig. 5 shows fringes obtained when illuminated by linearly polarized light for both single wedge and double wedge. These correspond to configurations $w$ and $w w^{\prime}$ defined in Table 1, though the wedges are not compounded in this test. The fringes are clearly visible when polarized light enters the spectrograph, and are not visible when unpolarized light is used. A more formal laboratory validation follows below in Sec. 6 . 


\section{Data analysis Methods}

In equations (1)-(3), (5), and (6), the Stokes parameters are coefficients of orthogonal trigonometric functions. While Fourier methods could be used to retrieve these coefficients, we prefer a linear least squares solution. This enables us to generate formal error estimates, in addition to providing the Stokes coefficients in a straightforward fashion. With a least squares approach, one can also take the dispersion of birefringence into account in a more straightforward way than with Fourier methods. The general methods are described in the Appendix. If the spatial profile at a fixed wavelength is given by

$$
I_{\mathrm{obs}}=I i_{c}+Q q_{c}+U u_{c}+V v_{c}
$$

for a single beam, or

$$
I^{\prime}=\left(I_{\|}-I_{\perp}\right) /\left(I_{\|}+I_{\perp}\right)=q \cdot q_{c}+u \cdot u_{c}+v \cdot v_{c}
$$

for the dual beam formalism, then the terms $i_{c}, q_{c}, u_{c}$ and $v_{c}$ are trigonometric functions whose coefficients are the Stokes parameters we seek, i.e., Eqs. (1)-(3), (5), (6), and others below. Since these trigonometric functions take on a set of known values at each $x_{i}$ along the profile, standard methods can be applied to solve for their coefficients given an observed intensity profile $I_{\mathrm{obs}}\left(x_{i}\right)$ or $I^{\prime}\left(x_{i}\right)$, as described in the Appendix . The solution depends on the inverse of the curvature matrix, which is derived from products of the functions $i_{c}, q_{c}$, $u_{c}$ and $v_{c}$. The solution is the inverse of the curvature matrix, multiplied by a vector derived from the observed profile and the same set of functions. The covariance matrix is also the inverse of the curvature matrix, and uncertainties on the Stokes parameters are taken as the square root of the diagonal terms of that matrix.

Analytic expressions can be derived for the terms of the curvature matrix and its inverse. Replacing the summations by integrals over complete periods, it may be seen that in a formal mathematical sense the Stokes parameters are independent because the trigonometric functions are orthogonal for most of the methods described. That is, the integrals of their products become zero and the inverse of the curvature matrix is diagonal. Hence there is no formal covariance between the terms. In an instrumental realization this should translate to no formal cross-talk between the Stokes parameters. In the cases where there are off-diagonal terms in the curvature matrix inverse, we discuss this in the text.

The description so far is idealized, however. In general, summations are not over complete periods, and departures of the wedge characteristics from those assumed, e.g. the exact value of the slope, and sampling errors can all yield off-diagonal terms. We discuss methods to deal with such issues in Sec. 5. These terms can generally be included in the analytic analysis, although the equations can become complicated depending on which tolerances are explored. An example can be found at the end of Sec. 4.B.3. 
It will also be necessary to calibrate a given system. The goal of calibration can be either to determine the (hopefully) small correction factors to the analytic formulae, or else a completely empirical calibration approach can be adopted, as discussed in Sec. 5.B.

To within an order of magnitude, we see in the analytic solutions, presented in the Appendix, the expected $1 / \sqrt{N_{t o t}}$ dependence for the sensitivity to which polarization can be measured, where $N_{\text {tot }}$ is the number of detected photons.

\section{Concept for Full Stokes Polarimetry}

The use of precision circular spectropolarimetry as a biosignature [3] requires accurate, sensitive measurement of the circular polarization spectrum. It is strongly preferred that all Stokes parameters be measured in order to better understand the physics involved and to guard against instrumental cross-talk, where one Stokes parameter is measured incorrectly, through instrumental effects, as another. Here, we describe two options to acquire full Stokes polarimetry data. The first, briefly described, is a brute force approach, where we simply place two versions of the configurations described above, next to one another. The second approach is to use an additional wedge or compound wedge with different fast axes and wedge angles to fully encode all Stokes parameters on a single data frame using a single optical bench.

\section{A. Two spectrograph slits}

In Secs. 2A and 2B, Eqs. (1) - (6), we showed how to encode two Stokes parameters simultaneously. By dispensing with the quarter wave retarder in those configurations, e.g. using a single wedge single beam, we have

$$
I_{Q}=0.5(I+Q \cos \phi-V \sin \phi)
$$

If a second device is constructed with its wedge fast axis horizontal, $0^{\circ}$, and analyzer at $45^{\circ}$ to the horizontal, then we have

$$
I_{U}=0.5(I+U \cos \phi+V \sin \phi)
$$

These two configurations can be processed indendently, and typically $|V| \ll|Q|,|U|$. Following the formalism of Eq. (4), the influence of a component of circular polarization $v$ is to shift the phases of the spatially modulated profiles by amounts, in pixel space, of $\delta x=X \tan ^{-1}(v / q) /(2 \pi)$ for the first device and $\delta x=X \tan ^{-1}(v / u) /(2 \pi)$ for the second device, where $X$ is the spatial period corresponding to one wavelength of retardance. For small $v$, the spatial shift of the profile is therefore $\delta x \approx X v / 2 \pi q$ and $\delta x \approx X v / 2 \pi u$, respecively. For example, if the source is $3 \%$ polarized, $q=0.03$, if $v=10^{-3}(0.1 \%$ circularly polarized $)$, and if $X=10$ pixels, the spatial shift is 0.05 pixels. The accuracy to which the spatial shift 
can be determined depends in principle on the total number of photons rather than the value of $X$, provided there are sufficient points within $X$ to properly sample the profile (see Appendix). Systematic instrumental effects are also likely to enter; however, measurement to a precision of better than 0.01 pixels can be achieved in precision astrometry, and these accuracies ought to be feasible.

\section{B. Double wedges}

A concise solution to acquiring full Stokes spectropolarimetry may be obtained by using a more complex group of birefringent wedges. Our technique is analogous to the dual PEM polarimeters which encode the different Stokes parameters using different carrier frequencies. If we follow the first wedge or compound wedge by a second wedge that has twice the thickness gradient, and a fast axis oriented $45^{\circ}$ to the first one, then the resultant intensity profiles encode the full Stokes information. The trigonometric functions involved are more complex than the simple sine and cosines of above, but they are relatively straightforward and still orthogonal. This configuration has the advantage of measuring the full Stokes parameters completely and simultaneously for a source, without minor viewing perspective differences in the double slit option (Sec. 4.A) and without needing to combine measurements from two essentially independent polarimeters.

We describe three versions of this concept, though it is clear that a variety of permutations and options are available under the umbrella of this general approach. The first two versions, to illustrate the principles, are to place a single wedge with twice the thickness gradient parallel and antiparallel, respectively, after a single wedge oriented as for the linear polarimetry application above. We conclude with a discussion of a double compound wedge, in which the second pair has twice the thickness gradient of the first. No quarter wave retarder is required. An example of a different permutation would be to reverse these two compound wedge pairs, which would result in a potentially more convenient choice of analyzer angle following the wedges. We defer discussion of such an option to a later paper and focus here on providing a proof of concept and demonstration of the approach. The naming convention used in the following subsection headings is described in the footnote to Table 1, which summarizes the terms in the equations for the amplitude modulation. Table 2 presents formulae for the uncertainties on the normalized Stokes parameters derived using the linear least squares methods of Sec. 3 and the Appendix. Table 3 gives the error estimates for the unnormalized Stokes parameters. Throughout, we assume standard $(1 \sigma)$, coverage factor $k=1$, uncertainties.

\section{B.1. Two individual parallel wedges: $w W$}

In this version we assume the first wedge has a birefringence gradient, described by $\phi(x)$, and fast axis at $45^{\circ}$ to the horizontal, as above. We assume the second wedge has twice the 
retardance $2 \phi(x)$ at location $x$ and has fast axis aligned with the slit, $0^{\circ}$. Additionally, it is necessary to allow the analyzer angle to be set at angles other than zero degrees, in order not to lose dependence to the Stokes $U$ parameter. We define the additional variable $\theta$ as the angle of the transmission axis of the analyzer with respect to the horizontal or slit direction. In the dual beam configuration, the transmission axis of the second polarized beam is $\theta+90^{\circ}$. We retain the labelling $I_{\|}$and $I_{\perp}$ for these two beams, respectively. In practice, there are likely to be constant offsets and a gradient of the second wedge not exactly twice that of the first wedge. These terms can generally be included in the analytic analysis of Sec. 3, but for clarity in introducing the concepts, we set them aside for now. It can be shown that the amplitude modulated profiles for this configuration are, for the single beam:

$$
\begin{array}{r}
I_{\|}=0.5(I+Q(\cos \phi \cos 2 \theta+\sin \phi \sin 2 \phi \sin 2 \theta)+U \cos 2 \phi \sin 2 \theta \\
+V(\cos \phi \sin 2 \phi \sin 2 \theta-\sin \phi \cos 2 \theta)), \\
I_{\perp}=0.5(I-Q(\cos \phi \cos 2 \theta+\sin \phi \sin 2 \phi \sin 2 \theta)-U \cos 2 \phi \sin 2 \theta \\
-V(\cos \phi \sin 2 \phi \sin 2 \theta-\sin \phi \cos 2 \theta)),
\end{array}
$$

and hence for the dual beam,

$$
\begin{aligned}
I^{\prime}=q(\cos \phi \cos 2 \theta & +\sin \phi \sin 2 \phi \sin 2 \theta)+u \cos 2 \phi \sin 2 \theta \\
& +v(\cos \phi \sin 2 \phi \sin 2 \theta-\sin \phi \cos 2 \theta) .
\end{aligned}
$$

We see that if $\theta=0^{\circ}$, then the coefficient of $U$ is zero and hence we cannot derive the value of $U$. There is no particular reason to have the analyzer at such an angle. For example, in Fig. 4 we use $\theta=45^{\circ}$ for the double wedge configurations. However, the derived variance for each of the Stokes parameters is a function of $\theta$. Depending on the application, it may be helpful to select $\theta$ to minimize the variance of the estimate for Stokes $V$, since Stokes $V$ is usually orders of magnitude smaller than the linear Stokes parameters. Below, Sec. 4.B.3 we show an example.

\section{B.2. Two individual antiparallel wedges: $w W^{\prime}$}

If the second wedge is placed in the opposite direction to the first, so as to minimize the geometric angles of the two wedges together, we have phase gradients of $\phi(x)$ and $(\zeta-2 \phi(x))$, respectively, for the two wedges, where $\zeta$ is a constant, presumed unknown. It can be shown that the amplitude modulated profiles for this configuration are, for the single beam:

$$
\begin{array}{r}
I_{\|}=0.5(I+Q(\cos \phi \cos 2 \theta+\sin \phi \sin (\zeta-2 \phi) \sin 2 \theta)+U \cos (\zeta-2 \phi) \sin 2 \theta \\
+V(\cos \phi \sin (\zeta-2 \phi) \sin 2 \theta-\sin \phi \cos 2 \theta)),
\end{array}
$$




$$
\begin{array}{r}
I_{\perp}=0.5(I-Q(\cos \phi \cos 2 \theta+\sin \phi \sin (\zeta-2 \phi) \sin 2 \theta)-U \cos (\zeta-2 \phi) \sin 2 \theta \\
-V(\cos \phi \sin (\zeta-2 \phi) \sin 2 \theta-\sin \phi \cos 2 \theta)),
\end{array}
$$

and for the dual beam

$$
\begin{array}{r}
I^{\prime}=q \cos \phi \cos 2 \theta+\sin \phi \\
\sin (\zeta-2 \phi) \sin 2 \theta)-u \cos (\zeta-2 \phi) \sin 2 \theta \\
-v(\cos \phi \sin (\zeta-2 \phi) \sin 2 \theta-\sin \phi \cos 2 \theta) .
\end{array}
$$

In this case, where the second wedge is configured to run antiparallel to the first one, there is an off-diagonal term in the inverse curvature matrix $\mathbf{B}^{-1}$ (defined in the Appendix) containing the term $(\sin \zeta \sin 4 \theta)$. (The same term appears if the wedges run parallel, but with a phase offset.) The inverse of the curvature matrix is the crucial mathematical entity in both solving for the Stokes parameters and estimating their variances. If the off-diagonal terms are zero then the trigonometric functions are orthogonal, and there are no formal dependencies of one Stokes parameter on the others or covariances between them. If there are off-diagonal terms, then there may be such dependencies and covariances.

The off-diagonal term can be set to zero if the analyzer is placed at an angle $\theta$ such that $\sin 4 \theta$ is zero. Since the $U$ coefficient also includes terms involving $\sin 2 \theta$, which we do not want to be zero, the desired analyzer angle to eliminate cross-dependencies is $\theta=45^{\circ}$. There may be a trade choice based on the optimization required, between making the off-diagonal terms of the inverse curvature matrix zero and minimizing the variance of a particular Stokes parameter, which can occur at a different analyzer angle.

\section{B.3. Two compound wedge pairs: $w w^{\prime} W W^{\prime}$}

By analogy with the compound double wedge in the linear polarimetry example, described in Sec. 2.B, it is possible to use two compound double wedges to provide zero retardance in the center of the optic. The first wedge pair has one gradient, as for the optic used in Sec. 2.B above ( $w w^{\prime}$ in the notation of Table 1). Each element of the second wedge pair has double the thickness gradient. The first has its fast axis along the slit, while the second has its fast axis orthogonal to it, so that the retardances are $\xi-2 \phi(x)$ and $\xi+2 \phi(x)$, respectively. Again, the compound device with four wedges is rectangular in shape. It can be shown that the amplitude modulated profiles for this configuration are, for the single beam,

$$
\begin{array}{r}
I_{\|}=0.5(I+Q(\cos 2 \phi \cos 2 \theta+\sin 2 \phi \sin 4 \phi \sin 2 \theta)+U \cos 4 \phi \sin 2 \theta \\
+V(\cos 2 \phi \sin 2 \phi \sin 2 \theta-\sin 2 \phi \cos 2 \theta)), \\
I_{\perp}=0.5(I-Q(\cos 2 \phi \cos 2 \theta+\sin 2 \phi \sin 4 \phi \sin 2 \theta)-U \cos 4 \phi \sin 2 \theta \\
-V(\cos 2 \phi \sin 2 \phi \sin 2 \theta-\sin 2 \phi \cos 2 \theta)),
\end{array}
$$


and for the dual beam

$$
\begin{array}{r}
I^{\prime}=q(\cos 2 \phi \cos 2 \theta+\sin 2 \phi \sin 4 \phi \sin 2 \theta)-u \cos 4 \phi \sin 2 \theta \\
-v(\cos 2 \phi \sin 4 \phi \sin 2 \theta-\sin 2 \phi \cos 2 \theta) .
\end{array}
$$

These functions are orthogonal to one another. Fig. 6 shows the derived variance as a function of the analyzer angle, with analytic formulae presented in Table 2. The minimum variance for Stokes $V$, which typically has the smallest value of the Stokes parameters, is given at analyzer angle with $\tan 4 \theta=-2$, which implies $\theta \approx 74.1^{\circ}$. The minimum variance for Stokes $Q$ is at $\tan 4 \theta=+2$, i.e., $\theta \approx 15.9^{\circ}$, and that for $U$ is at $\theta=45^{\circ}$. The value of the minimum variance is, for $q$ and $v, \sigma(q, v) \approx 1.24 / \sqrt{N_{t o t}}$, which represents the photon counting limit for such a device and which is quite close to the canonical $1 / \sqrt{N_{\text {tot }}}$ value.

If the centers of the two compound wedges are misaligned by a spatial distance $s$, then there is an additional term in the above equations, which we characterize by a phase offset in the second wedge pair. That is, their retardances are given by $\xi-2 \phi(x)-a$ and $\xi+2 \phi(x)+a$, respectivel, where $a=4 \pi s / X$. In this case, the amplitude modulation expressions are

$$
\begin{array}{r}
I_{\|}=0.5(I+Q(\cos 2 \phi \cos 2 \theta+\sin 2 \phi \sin (2 a+4 \phi) \sin 2 \theta)+U \cos (2 a+4 \phi) \sin 2 \theta \\
+V(\cos 2 \phi \sin (2 a+4 \phi) \sin 2 \theta-\sin 2 \phi \cos 2 \theta)), \\
I_{\perp}=0.5(I-Q(\cos 2 \phi \cos 2 \theta+\sin 2 \phi \sin (2 a+4 \phi) \sin 2 \theta)-U \cos (2 a+4 \phi) \sin 2 \theta \\
-V(\cos 2 \phi \sin (2 a+4 \phi) \sin 2 \theta-\sin 2 \phi \cos 2 \theta)),
\end{array}
$$

and for the dual beam

$$
\begin{array}{r}
I^{\prime}=q(\cos 2 \phi \cos 2 \theta+\sin 2 \phi \sin (2 a+4 \phi) \sin 2 \theta)-u \cos (2 a+4 \phi) \sin 2 \theta \\
-v(\cos 2 \phi \sin (2 a+4 \phi) \sin 2 \theta-\sin 2 \phi \cos 2 \theta) .
\end{array}
$$

Following through the least squares analysis, it can be seen that the miscentering introduces an off-diagonal term in the inverse of the curvature matrix, correlating the errors in $Q$ and $V$, and again involving $\sin 4 \theta$. Hence, this off-diagonal term can be set to zero by setting $\theta=45^{\circ}$. If this is not done, or the tolerances do not allow it, then the presence of a covariance term in and of itself does not bias the result. The random errors on $Q$ correlate with the errors on $V$. The actual values of $Q$ and $V$ do not correlate, though, provided that the covariance term is known. In principle the covariance term does contribute to the overall error budget, although assuming independence yields a good description of the actual observed variance in Monte Carlo simulations, shown in Fig. 7. In the case where the term is present, but known, we still correctly derive the Stokes parameters without cross-talk. 
Cross-talk occurs if the covariance is not characterized correctly. For the example here of two miscentered wedge pairs, if we characterize the miscentering by $a / X$, the ratio of the offcenter distance to the spatial distance corresponding to one wavelength in the first wedge pair, then the true value of Stokes $v$, say, is $v_{t r u e}=s y_{1}\left[B^{-1}\right]_{q v}+s y_{4}\left[B^{-1}\right]_{v v}$, following the notation of the Appendix. If we incorrectly derive $v_{e s t}=s y_{4}\left[B^{-1}\right]_{v v}$, ignoring the cross-term, then $v_{\text {true }}-v_{\text {est }}=s y_{1}\left[B^{-1}\right]_{q v}=q\left[B^{-1}\right]_{q v} /\left[B^{-1}\right]_{q q}$. A formal tolerance analysis can be carried out, and to completely ignore this term, while restricting the cross talk $<10^{-3}$, requires $\theta$ to be within $\lesssim 0.1^{\circ}$ of $45^{\circ}$ if the miscentering is such as to maximize the cross talk. If needed, there are two options to relax this constraint within the context of an analytical approach: (i) do not ignore the term! and (ii) increase the spatial scale $X$ to ease the requirement on $s / X$. Empirical calibration approaches are also possible as discussed below.

\section{Practical Implementation, Requirements, Sensitivities and Calibration}

Wedge retarders, such as those discussed here may be custom manufactured. However, testable quality versions are available off-the-shelf under the guise of depolarizers or scramblers. Quartz or calcite provide plausible birefringent materials. The ordinary and extraordinary refractive indices and birefringences are, for quartz, $n_{o}=1.5384, n_{e}=1.5473$, $n_{e}-n_{o}=0.0089$, respectively, and for calcite $n_{o}=1.647, n_{e}=1.480, n_{e}-n_{o}=-0.167$. For example, if the retarders have a wedge gradient of $3^{\circ}$, then at $500 \mathrm{~nm}$ the retardance increases by one wavelength over a distance $X$ of $\approx 1.1 \mathrm{~mm}$ for quartz and $0.06 \mathrm{~mm}$ for calcite. If these scales are projected 1:1 onto a detector with $5 \mu \mathrm{m}$ pixels, then these values correspond to $X \approx 214$ and 11 pixels for quartz and calcite, respectively.

\section{A. An empirical calibration approach}

In a similar fashion to the analysis described in Sec. 4.B.3, the least squares methods lend themselves to formal tolerance analyses, as well as parameter estimation. However, a comprehensive analysis of all plausibly relevant parameters is impractical and premature. Instead, we consider an alternative approach, which is purely empirical. For a very general set of optical component characteristics, the generic versions of the amplitude modulated intensity profile, Eqs. (7) and (8), are valid, even if the exact forms of the functions $i_{c}, q_{c}, u_{c}$ and $v_{c}$ are not known. If we present the system in turn with unpolarized light, and then $100 \%$ polarized light oriented in the $Q$ direction, the $U$ direction, and finally, with $100 \%$ circularly polarized light, then the empirical response gives the functions $i_{c}, q_{c}, u_{c}$ and $v_{c}$. These empirically derived functions can then be used to numerically derive the curvature matrix and its inverse. As in Sec. 4.B.3, the presence of covariance terms in the matrices by itself does not invalidate the approach, because, in principle, with a high quality set of calibration observations (which would appear much like the examples shown in Fig. 4), they are implicitly 
known. It is only when the terms are not correctly accounted for, that problems may arise; that is, if the calibration sources are not of high enough quality. It is also likely that the variance will be a function of analyzer angle, as above. Hence empirical versions of Fig. 7 may be useful to explore trade space.

We expect, in general, the empirical calibration method to be a very important approach, potentially offering the best strategy for deriving the Stokes parameters accurately. However it remains to be seen to what degree the required tolerances, or calibration stability, limit the performance of these devices in practice. Our intent is to develop additional laboratory experience to understand these issues.

\section{B. Sensitivities}

Component birefringence will depend on temperature. This can be mitigated by (i) stabilizing the temperature, (ii) continuous calibration, and (iii) compounding carefully chosen materials. To obtain an idea of the order of magnitude of the temperature sensitivity, we use the temperature dependent formula for the birefringence $B=n_{e}-n_{o}$ of quartz, given by [21], which yields $(d B / d T) / B \approx 1.2 \times 10^{-4}$ for the range $T=0{ }^{\circ} \mathrm{C}$ to $25^{\circ} \mathrm{C}$. Since the wedge should keep its shape under expansion or contraction, only the birefringence term matters and imprints itself as an identical fractional change $\epsilon=1.2 \times 10^{-4} d T$ on the spatial wavelength $X$. It can be shown that the resulting fractional change in $q$ for the dual beam single wedge example is one half the fractional change in $B$, for an input beam consisting of purely $q$, and the spurious cross-talk $u^{\prime}$ into the other Stokes parameter $u$ is $u^{\prime}=\pi \epsilon q$. Hence a $1{ }^{\circ} \mathrm{C}$ temperature change would result in a spurious polarization (cross talk) of $u^{\prime} \approx 3 \times 10^{-5}$ for a $10 \%$ linearly polarized source.

The presence of two refractive indices in a wedge-shaped optic will cause a prismatic separation of the orthogonally polarized beams. The magnitude of this effect will depend on the details of the optical system designed. In our laboratory testing, this issue was not significant.

If the beam incident on a single wedge has significant convergence, then the retardance seen by light at different angles differs by approximately $1 / \cos \zeta$ where $\zeta$ is the angle to the normal. For this retardance difference to be less than $\lambda / 10$, say, the $f$-number of the beam needs to be slower than $f / 9$ for a $1 \mathrm{~mm}$ thickness quartz and $f / 40$ for $1 \mathrm{~mm}$ thickness calcite. The convergence requirement scales as the inverse square root of the thickness.

We carried out a number of Monte-Carlo simulations and found that the retrieved solutions are close to the theoretical expressions given in the tables provided that (i) at least one full period is sampled and (ii) there are sufficent sampling points within the full cycle to properly sample the highest frequency component.

In cases where the fringes have significant slope, as described above, it will be necessary 
for the spectral resolution to be sufficient to separate the fringes. If we require the retardance change $\Delta \phi /(2 \pi)<0.1$, this is $(\partial \phi / \partial \lambda) \Delta \lambda<0.1$, where $\phi=2 \pi L\left(n_{e}-n_{o}\right) / \lambda, L$ the thickness. Hence the required spectral resolution is $R \equiv \frac{\lambda}{\Delta \lambda}=10 L\left(n_{e}-n_{o}\right) / \lambda$. For $2 \mathrm{~mm}$ thick quartz this is $R>360$ and for $1 \mathrm{~mm}$ calcite, $R>3330$. For the compounded wedge optics which straighten the fringes, this constraint is relaxed to the point of being essentially irrelevant.

\section{Laboratory Validation}

We established an optical test bench to allow us to provide an empirical proof of concept for the approach presented in this paper, and to demonstrate that the device functions as a polarimeter. The optical test bench is shown and illustrated in Fig. 8.

Light sources, either halogen continuum white light, or a variety of line lamps for wavelength calibration, illuminated an integrating sphere's entrance port. The light emerging from a separate port at right angles to the first is expected to be unpolarized. However, to ensure an unpolarized source and to provide a uniform location for our measurements, the emergent light was directed to fall onto an opal diffusing screen. Following the opal screen, and located close to the screen, the light optionally encountered polarizing elements $(Q, U$, or $V$ ) for calibration, samples to measure the polarized transmission spectrum, or nothing, to provide an intensity reference spectrum.

The spectrograph consisted of a slit, a collimator, a transmission grating, and a camera. The slit was $125 \mu \mathrm{m}$ by $1 \mathrm{~cm}$ and was located at one focus of an $f / 1.450 \mathrm{~mm}$ Nikon collimating lens. ${ }^{1}$ The transmission diffraction grating was ThorLabs Part \# GT50-06V with 600 grooves $/ \mathrm{mm}$. A second f/1.4 $50 \mathrm{~mm}$ Nikon lens imaged the spectrum onto a Quantum Scientific Imaging 6838 Mpix cooled CCD Camera with $3326 \times 2504$ pixels of size 5.4 micron.

For the polarization analyzer, we placed a Meadowlark precision linear polarizer, DPM100VIS between the spectrograph slit and the collimating lens, using a rotary mount to allow adjustment of the analyzer angle. The extinction ratio across the visible spectrum for this polarizer is $\sim 10^{4}$, and exceeds 100:1 from approximately $375 \mathrm{~nm}$ to $725 \mathrm{~nm}$. The birefringent wedges were placed on a platform next to the spectrograph slit between the slit and the source (i.e. before the light enters the spectrograph). The optional quarter wave retarder was mounted, when used, between these wedges and the source and was an achromatic Meadowlark quarter wave retarder AQM-100-545, which is effective between $450 \mathrm{~nm}$ and $630 \mathrm{~nm}$. The birefringent wedges themselves were a mix of customized and off-the-shelf quartz scramblers from Karl-Lambrecht Corporation. Off-the-shelf wedges had a pitch angle

\footnotetext{
${ }^{1}$ Certain commercial equipment, instruments, or materials are identified in this paper in order to specify the experimental procedure adequately. Such identification is not intended to imply recommendation or endorsement by the Space Telescope Science Institute, the National Institute of Standards and Technology, or the Universiteit Leiden nor is it intended to imply that the materials or equipment identified are necessarily the best available for the purpose.
} 
of $\approx 3^{\circ}$, and the customized pieces used a pitch angle of $\approx 6^{\circ}$.

The distance between the source and the spectrograph slit was approximately $0.6 \mathrm{~m}$, and the entire system was contained within a series of light-tight baffles and boxes to eliminate stray light. Locating the birefringent wedges externally to the spectrograph not only allows us to ignore possible polarization due to the slit, but also allowed easy access for switching configurations, and permitted us to focus the spectrograph onto a single slit position.

The first exercise was to attempt to reproduce the appearance of the theoretical data frames for the various configurations presented in Fig. 4. Fig. 9 shows the results. To do this, we used the white halogen continuum source in combination with linearly and circularly polarizing filters. For the first four configurations (first four columns in Fig. 9), we used a set of $60 \mathrm{~mm}$ astronomical polarizing filters that utilize HN38 Polaroid mounted in a magnesium fluoride substrate to approximate 100\% linearly polarized light (first two rows of the first four columns in Fig. 9). We used a cholesteric liquid crystal technology (CLC) filter to approximate 100\% circularly polarized light (third row of the first four columns in Fig. 9). For the final configuration (fifth column of Fig. 9), we used a polarization state generator that utilized a precision linear polarizer in combination with a Fresnel rhomb [22] that is capable of producing close to $100 \%$ polarized light anywhere on the Poincaré sphere. We also obtained data frames without any polarizing optics to provide a "flat-field" reference. The spectral scale was wavelength calibrated using argon and mercury line lamps. We defined the slit direction to correspond to $Q$ and $45^{\circ}$ to the slit to correspond to $U$.

By comparing Figs. 4 and 9, it is apparent that the empirical data reproduce the qualitative expectations extremely well. Differences in detail can be attributed to different absolute thicknesses of the wedges (in practice the $3^{\circ}$ and $6^{\circ}$ wedges had very different thicknesses) relative to one another and to the theoretical model, and to essentially random centering of the crossover points for wedge pairs relative to the slit and to one another. Care was taken with the sign convention and parity of the wedges to reproduce the directions for fast axes and wedge gradients used in the models. The empirical wavelength range shown corresponds to $550 \mathrm{~nm}$ to $700 \mathrm{~nm}$, and the slit height to $2 \mathrm{~mm}$ in Fig. 9. We consider the data obtained in this exercise to be fully consistent with the theoretical expectations, given the practical uncertainties described.

The second exercise was to test the linear polarization mode described in Sec. $2 \mathrm{~B}$. We placed a compound $3^{\circ}$ wedge pair with the fast axes crossed, running corner to corner at $\pm 45^{\circ}$ to the spectrograph slit, in front of the spectrograph slit. An achromatic quarter wave retarder was placed upstream of the wedge pair, with its fast axis oriented $0^{\circ}$ to the slit. Calibration measurements were taken using the standard suite of polarizing filters, and the resulting data frames were used to derive empirical "coefficient" frames for use in the leastsquares retrieval procedure of Sec. 3 and Sec. 5A, with additional theoretical analysis in 
the Appendix. We placed an Schott BG18 colored glass filter (broad bandpass with peak transmission near $\sim 510 \mathrm{~nm}$ ) close to the source and orthogonal to the beam and took a single data frame. We then rotated the filter about the vertical axis by approximately $30^{\circ}$ and took a second data frame. Using these single data frames in conjunction with the procedures described in Sec. 3 and Sec. 5A, we derived polarization and intensity spectra, shown in Fig. 10. The average linear polarization for the region $500 \mathrm{~nm}$ to $550 \mathrm{~nm}$ was $0.39 \%$ and $6.2 \%$ with standard deviations $0.02 \%$ and $0.35 \%$ respectively. For a uniform glass sheet of refractive index $n=1.5$ in air, we expect transmitted light to be polarized $6.3 \%$ for an incident angle of $30^{\circ}$. We emphasize that these results were obtained using a single data frame with no moving parts once the calibration data had been acquired.

The third exercise was to demonstrate our ability to obtain full Stokes polarimetry from a single data frame. For this, we used the compound $3^{\circ}$ wedge pair used in the previous exercise, followed by a compound $6^{\circ}$ wedge pair with fast axes at $0^{\circ}$ and $90^{\circ}$. We removed the quarter wave retarder. As an interesting source of circularly polarized light, we used a

pair of plastic 3D cinema glasses. These glasses comprise a quarter wave sheet and polarizing sheet in combination. Used as viewers, they either transmit or extinguish circularly polarized light, depending on its sign. But, in reverse, they produce circularly polarized light of opposite signs for each "eye." We took single data frames through each eye in turn and processed the data frames according to the methodology of Sec. 3 and Sec. 5A, using the empirical suite of calibration data frames. The results are shown in Fig. 11. The method produced excellent results on these sources, yielding the expected extremely high level of polarization, with opposite sign for the two eyes. We also show the derived linear polarization, to show that we are also measuring full the Stokes vector in these single data frames.

We defer attempts to carry out precision polarimetry given the rudimentary nature of our optical bench coupled to imperfect calibration source availability. However, we believe that these preliminary results are extremely encouraging and satisfy our desire to provide a proof of concept in the laboratory and to demonstrate that the desired polarimetric information can be retrieved from single data frames.

\section{Conclusions}

We have described an approach to polarization measurement that uses no moving parts and that relies on simple, robust optical components. Either linear polarimetry or full Stokes polarimetry can be carried out. The method depends on the use of an area detector, such as a CCD, with the light spread across a region of the detector. If the system can be made photon-limited in sensitivity, this spreading of the light improves the polarimetry, since typical CCD well-depths are only of order $10^{5}$. With modest spreading of the light, a single photon limited frame should be able to reach precision of order $10^{-4}$ in polarization. The 
influence of departures from ideal circumstances still remains largely to be explored. Hence, we do not know at this stage whether this approach will be able to achieve extremely high accuracy. However the robustness and simplicity of the components involved offers cause for optimism. Other approaches, such as the spectral modulation method for linear polarimetry [5], offer alternative methods for static polarimetry in hostile environments. Compared to that approach, the methods presented here yield a cleaner separation of the spectroscopy and polarimetry, at the expense of additional detector surface area requirements. The methods may be applied in the UV or IR as well as in the visible wavelength range.

Since the entire polarization information is contained within a single data frame, the method is well-suited to measuring the polarization of transient sources and scenes where the polarimeter and target are in rapid relative motion. Since the optics are robust, simple and require no moving parts, we anticipate that these methods will prove useful for application in space.

Acknowledgments: We acknowledge support from the STScI JWST Director's Discretionary Research Fund JDF grant number D0101.90152. STScI is operated by the Association for Universities for Research in Astronomy, Inc., under NASA contract NAS5-26555. Patent pending, all rights reserved.

\section{Appendix: General Linear Least Squares Methods}

We follow Bevington $[23,24]$ and let the general problem to be solved be

$$
y\left(x_{i}\right)=a i_{c}\left(x_{i}\right)+b q_{c}\left(x_{i}\right)+c u_{c}\left(x_{i}\right)+d v_{c}\left(x_{i}\right),
$$

where measurements $y_{i}$, either the intensity $I$ in the single beam case or $\left(I_{\|}-I_{\perp}\right) /\left(I_{\|}+I_{\perp}\right)$ in the dual beam case, are made at points $x_{i}$ and $y_{i}=y\left(x_{i}\right)+\epsilon_{i}$, with $y$ the true underlying value and $\epsilon_{i}$ its error (assumed random, independent) at location $x_{i}$. The terms $i_{c}, q_{c}, u_{c}$, and $v_{c}$ are trigonometric functions that encode the Stokes parameters $I, Q, U$, and $V$ or $q$, $u$, and $v$, and their coefficients $a, b, c$, and $d$ are the Stokes parameters to be derived. The mapping and specific functions depend on the chosen configuration, but all configurations discussed here can be expressed in this way. Sometimes the functions are identically zero, implying no sensitivity to that parameter. 
The $\chi^{2}$ function is then

$$
\chi^{2}=\sum_{i=1}^{N} \epsilon_{i}^{2} / \sigma_{i}^{2}=\sum_{i=1}^{N} \frac{1}{\sigma_{i}^{2}}\left[y_{i}-y\left(x_{i}\right)\right]^{2}=\sum_{i=1}^{N} \frac{1}{\sigma_{i}^{2}}\left[y_{i}-a i_{c}\left(x_{i}\right)-b q_{c}\left(x_{i}\right)-c u_{c}\left(x_{i}\right)-d v_{c}\left(x_{i}\right)\right]^{2},
$$

and to solve, we set the partial derivatives of $\chi^{2}$ with respect to each of $a, b, c$, and $d$ equal to zero:

$$
\begin{aligned}
& \frac{\partial \chi^{2}}{\partial a}=0=-2 \sum \frac{1}{\sigma_{i}^{2}} i_{c}\left(y_{i}-a i_{c}-b q_{c}-c u_{c}-d v_{c}\right), \\
& \frac{\partial \chi^{2}}{\partial b} \quad=0=-2 \sum \frac{1}{\sigma_{i}^{2}} q_{c}\left(y_{i}-a i_{c}-b q_{c}-c u_{c}-d v_{c}\right), \\
& \frac{\partial \chi^{2}}{\partial c}=0=-2 \sum \frac{1}{\sigma_{i}^{2}} u_{c}\left(y_{i}-a i_{c}-b q_{c}-c u_{c}-d v_{c}\right), \\
& \frac{\partial \chi^{2}}{\partial d} \quad=0=-2 \sum \frac{1}{\sigma_{i}^{2}} v_{c}\left(y_{i}-a i_{c}-b q_{c}-c u_{c}-d v_{c}\right) .
\end{aligned}
$$

We require the curvature matrix $\mathbf{B}$ and summation vector $s_{y}$,

$$
\begin{gathered}
\mathbf{B} \equiv\left(\begin{array}{cccc}
\sum \frac{1}{\sigma_{i}^{2}} i_{c}^{2} & \sum \frac{1}{\sigma_{i}^{2}} i_{c} q_{c} & \sum \frac{1}{\sigma_{i}^{2}} i_{c} u_{c} & \sum \frac{1}{\sigma_{i}^{2}} i_{c} v_{c} \\
\sum \frac{1}{\sigma_{i}^{2}} i_{c} q_{c} & \sum \frac{1}{\sigma_{i}^{2}} q_{c}^{2} & \sum \frac{1}{\sigma_{i}^{2}} q_{c} u_{c} & \sum \frac{1}{\sigma_{i}^{2}} q_{c} v_{c} \\
\sum \frac{1}{\sigma_{i}^{2}} i_{c} u_{c} & \sum \frac{1}{\sigma_{i}^{2}} q_{c} u_{c} & \sum \frac{1}{\sigma_{i}^{2}} u_{c}^{2} & \sum \frac{1}{\sigma_{i}^{2}} u_{c} v_{c} \\
\sum \frac{1}{\sigma_{i}^{2}} i_{c} v_{c} & \sum \frac{1}{\sigma_{i}^{2}} q_{c} v_{c} & \sum \frac{1}{\sigma_{i}^{2}} u_{c} v_{c} & \sum \frac{1}{\sigma_{i}^{2}} v_{c}^{2}
\end{array}\right), \\
s_{y} \equiv\left(\sum \frac{1}{\sigma_{i}^{2}} i_{c} y_{i}, \sum \frac{1}{\sigma_{i}^{2}} q_{c} y_{i}, \sum \frac{1}{\sigma_{i}^{2}} u_{c} y_{i}, \sum \frac{1}{\sigma_{i}^{2}} v_{c} y_{i}\right),
\end{gathered}
$$

, respectively. With this terminology, the least squares equations become

$$
s_{y}=\mathbf{B} \cdot\left(\begin{array}{l}
a \\
b \\
c \\
d
\end{array}\right) .
$$

We solve for the vector $(a, b, c, d)$,

$$
\mathbf{a}=(a, b, c, d)=\mathbf{B}^{-1} \cdot s_{y} .
$$

Following standard procedures, e.g. [23], [24], ignoring covariances, the uncertainties on these parameters are

$$
\sigma_{a_{i}}^{2}=B_{i i}^{-1}
$$

where $a_{i}$ represents $a, b, c$, or $d$, and $\left[B^{-1}\right]_{i i}$ is the corresponding diagonal term of $\mathbf{B}^{-1}$. 
Our application is precision polarimetry, for which it is presumed (i) the degree of polarization is small, and (ii) light levels are relatively high. Hence, the intensity across the spatial segment is approximately constant and obeys Poisson counting statistics. That is, we assume the uncertainty is the same for each bin, $\sigma_{i}=\sigma=\left(N_{\gamma} / n x\right)^{(1 / 2)}$ where $N_{\gamma}$ is the total number of detected photons, and $n x$ is the number of bins across which the photons are distributed, i.e., the number of $x$ sampling points. For cases where the trigonometric functions that are embodied by $i_{c}, q_{c}, u_{c}, v_{c}$ are orthogonal (we approximate the summations by integrals over integer numbers of periods), $\mathbf{B}$ is diagonal. Hence its inverse is also diagonal, and the Stokes parameter solutions are independent of one another.

We choose as a simple example the single wedge with its fast axis at $45^{\circ}$ to the slit direction, which, in turn, defines the direction for Stokes $Q$. In general, we use Mueller matrix algebra to solve for the system. As in Sec. 2.A above, Eq. (1) gives the expression for the intensity at points $x_{i}: y_{i} \equiv I\left(x_{i}\right)=0.5\left(I-Q \cos \phi_{1}-U \sin \sin \phi_{i}\right)$ where $\phi_{i}=2 \pi\left(x_{i} / X\right)$. In the formalism above, $y_{i}=a i_{c}\left(x_{i}\right)+b q_{c}\left(x_{i}\right)+c u_{c}\left(x_{i}\right)+d v_{c}\left(x_{i}\right)$ so $i_{c}\left(x_{i}\right)=0.5, q_{c}\left(x_{i}\right)=0.5 \cos \left(2 \pi x_{i} / X\right)$, $u_{c}\left(x_{i}\right)=0.5 \sin \left(2 \pi x_{i} / X\right)$ and $v_{c}\left(x_{i}\right)=0$. In the absence of $V$ the matrix $\mathbf{B}$ is reduced to the $3 \times 3$ matrix

$$
\mathbf{B} \equiv \frac{1}{\sigma^{2}}\left(\begin{array}{ccc}
\sum i_{c}^{2} & \sum i_{c} q_{c} & \sum i_{c} u_{c} \\
\sum i_{c} q_{c} & \sum q_{c}^{2} & \sum q_{c} u_{c} \\
\sum i_{c} u_{c} & \sum q_{c} u_{c} & \sum u_{c}^{2}
\end{array}\right)
$$

The summations run across $n x$ pixels. Applying the expressions for the $q_{c}, u_{c}$, and $v_{c}$, we have

$\mathbf{B}=\frac{1}{4 \sigma^{2}}\left(\begin{array}{ccc}n x & \sum \cos \left(2 \pi x_{i} / X\right) & \sum \sin \left(2 \pi x_{i} / X\right) \\ \sum \cos \left(2 \pi x_{i} / X\right) & \sum \cos ^{2}\left(2 \pi x_{i} / X\right) & \sum \cos \left(2 \pi x_{i} / X\right) \sin \left(2 \pi x_{i} / X\right) \\ \sum \sin \left(2 \pi x_{i} / X\right) & \sum \cos \left(2 \pi x_{i} / X\right) \sin \left(2 \pi x_{i} / X\right) & \sum \sin ^{2}\left(2 \pi x_{i} / X\right)\end{array}\right)$.

We assume the summations cover an integer number of periods and approximate the sums using integrals, using $\int_{0}^{X} f(x) d x \approx \Delta x \sum_{1}^{n x} f\left(x_{i}\right)$, where $\Delta x=X / n x$ (the width of a bin in $x)$. Hence, $\sum f\left(x_{i}\right) \approx \frac{n x}{X} \int f(x) d x$. Thus, it can be shown for this example that

$$
\mathbf{B}=\frac{n x}{8 \sigma^{2}}\left(\begin{array}{lll}
2 & 0 & 0 \\
0 & 1 & 0 \\
0 & 0 & 1
\end{array}\right)
$$

and

$$
\mathbf{B}^{-1}=\frac{4 \sigma^{2}}{n x}\left(\begin{array}{lll}
1 & 0 & 0 \\
0 & 2 & 0 \\
0 & 0 & 2
\end{array}\right) .
$$

Now, we can go back to the expression for $s_{y}$ and solve for the Stokes parameters, noting that $\sum y_{i}=N_{\text {tot }}$, the total number of photons collected, and $s_{y}=$ 
$\frac{1}{2 \sigma^{2}}\left[\sum y_{i}, \sum \cos \left(2 \pi x_{i} / X\right) y_{i}, \sum \sin \left(2 \pi x_{i} / X\right) y_{i}\right]$, omitting the zero $V$ term. Hence the solutions from $(a, b, c, d)=\mathbf{B}^{-1} \cdot s_{y}$ are

$$
\begin{aligned}
& I \equiv a \quad=\left(\frac{N_{t o t}}{2 \sigma^{2}}\right)\left(\frac{4 \sigma^{2}}{n x}\right)=\frac{2 N_{t o t}}{n x}=2\langle y\rangle \\
& Q \equiv b \quad=\left(\frac{4}{n x}\right) \sum y_{i} \cos \left(2 \pi x_{i} / X\right) \\
& U \equiv c \quad=\left(\frac{4}{n x}\right) \sum y_{i} \sin \left(2 \pi x_{i} / X\right)
\end{aligned}
$$

Similarly, we can derive the uncertainties of the Stokes parameters. The uncertainty $\sigma$ is given by $\sigma^{2}=N_{\text {tot }} / n x$ and is assumed to be constant. Hence, reading directly from the expression for $\mathbf{B}^{-1}$, we have

$$
\begin{gathered}
\sigma(I) \equiv \sigma_{a}=\frac{2 \sqrt{N_{t o t}}}{n x} \\
\sigma(Q) \equiv \sigma_{b}=\frac{2 \sqrt{2 N_{t o t}}}{n x} \\
\sigma(U) \equiv \sigma_{c}=\frac{2 \sqrt{2 N_{t o t}}}{n x} .
\end{gathered}
$$

The uncertainties in the normalized Stokes parameters $q$ and $u$ are

$$
\sigma(q)=\sigma(u)=\sqrt{\frac{2}{N_{t o t}}} .
$$

Ignoring bias terms, it follows that the uncertainty on the degree of polarization is

$$
\sigma(p)=\sqrt{\frac{2}{N_{t o t}}} .
$$

The expressions for the trigonometric functions $i_{c}, q_{c}, u_{c}, v_{c}$ depend on the configuration and whether a dual beam formalism is adopted or not. We derived the expressions for these functions using Mueller matrix algebra for a selection of configurations, as presented in Table 1. In a similar fashion to this example, though with more complex manipulations, we can analytically invert the corresponding curvature matrix to derive both the solution and the uncertainty estimates, taking only the diagonal terms as the uncertainty. There are cases where the off-diagonal terms of $\mathbf{B}$ are non-zero, as discussed in the text. 
Table 1. Coefficients of Stokes parameters for different wedge configurations

\begin{tabular}{|c|c|c|c|c|c|}
\hline Wedges $^{\mathrm{a}}$ & Beam & $i_{c}$ & $q_{c}$ & $u_{c}$ & $v_{c}$ \\
\hline$q w$ & single & 0.5 & $0.5 \cos \phi$ & $0.5 \sin \phi$ & \\
\hline & dual & & $\cos \phi$ & $\sin \phi$ & \\
\hline$q w w^{\prime}$ & single & 0.5 & $0.5 \cos 2 \phi$ & $0.5 \sin 2 \phi$ & \\
\hline & dual & & $\cos 2 \phi$ & $\sin 2 \phi$ & \\
\hline$w W$ & single & 0.5 & $0.5(\cos \phi \cos 2 \theta+\sin \phi \sin 2 \phi \sin 2 \theta)$ & $0.5 \cos 2 \phi \sin 2 \theta$ & $0.5(\cos \phi \sin 2 \phi \sin 2 \theta-\sin \phi \cos 2 \theta)$ \\
\hline & dual & & $\cos \phi \cos 2 \theta+\sin \phi \sin 2 \phi \sin 2 \theta$ & $\cos 2 \phi \sin 2 \theta$ & $\cos \phi \sin 2 \phi \sin 2 \theta-\sin \phi \cos 2 \theta$ \\
\hline$w W^{\prime}$ & $\begin{array}{l}\text { single } \\
\text { dual }\end{array}$ & 0.5 & $\begin{array}{r}0.5(\cos \phi \cos 2 \theta+\sin \phi \sin (\zeta-2 \phi) \sin 2 \theta) \\
\cos \phi \cos 2 \theta+\sin \phi \sin (\zeta-2 \phi) \sin 2 \theta\end{array}$ & $\begin{array}{r}0.5 \cos (\zeta-2 \phi) \sin 2 \theta \\
\cos (\zeta-2 \phi) \sin 2 \theta\end{array}$ & $\begin{array}{r}0.5(\cos \phi \sin (\zeta-2 \phi) \sin 2 \theta-\sin \phi \cos 2 \theta) \\
\cos \phi \sin (\zeta-2 \phi) \sin 2 \theta-\sin \phi \cos 2 \theta\end{array}$ \\
\hline$w w^{\prime} W W^{\prime}$ & $\begin{array}{l}\text { single } \\
\text { dual }\end{array}$ & 0.5 & $\begin{array}{r}0.5(\cos 2 \phi \cos 2 \theta+\sin 2 \phi \sin 4 \phi \sin 2 \theta) \\
\cos 2 \phi \cos 2 \theta+\sin 2 \phi \sin 4 \phi \sin 2 \theta\end{array}$ & $\begin{array}{r}0.5 \cos 4 \phi \sin 2 \theta \\
\cos 4 \phi \sin 2 \theta\end{array}$ & $\begin{array}{r}0.5(\cos 2 \phi \sin 4 \phi \sin 2 \theta-\sin 2 \phi \cos 2 \theta) \\
\cos 2 \phi \sin 4 \phi \sin 2 \theta-\sin 2 \phi \cos 2 \theta\end{array}$ \\
\hline
\end{tabular}

${ }^{a}$ Notation for wedge configurations: $q$ denotes optional quarter wave retarder, $w$ denotes thickness gradient $\phi_{w}=2 \pi x / X$ and $W$ denotes twice the thickness gradient $\phi_{W}=4 \pi x / X$. A primed symbol denotes antiparallel wedge direction relative to unprimed. Wedges $w$ have fast axis at $45^{\circ}$ to the slit, $w^{\prime}$ at $-45^{\circ}$; wedges $W$ have fast axis at $0^{\circ}$, except in combination $W W^{\prime}$, when they are at $0 \circ$ and $90^{\circ}$ respectively.

Table 2. Error estimates for normalized Stokes parameters for different wedge configurations

\begin{tabular}{|c|c|c|c|c|}
\hline Wedges & Beam & $\sigma(q)$ & $\sigma(u)$ & $\sigma(v)$ \\
\hline \multirow[t]{2}{*}{$q w$} & single & $\left(2 / N_{t o t}\right)^{1 / 2}$ & $\left(2 / N_{t o t}\right)^{1 / 2}$ & \\
\hline & dual & $\left(2 / N_{t o t}\right)^{1 / 2}$ & $\left(2 / N_{t o t}\right)^{1 / 2}$ & \\
\hline \multirow[t]{2}{*}{$q w w^{\prime}$} & single & $\left(2 / N_{t o t}\right)^{1 / 2}$ & $\left(2 / N_{t o t}\right)^{1 / 2}$ & \\
\hline & dual & $\left(2 / N_{t o t}\right)^{1 / 2}$ & $\left(2 / N_{t o t}\right)^{1 / 2}$ & \\
\hline \multirow[t]{2}{*}{$w W$} & single & $2\left(2 / N_{t o t}\right)^{1 / 2}(3+\cos 4 \theta+2 \sin 4 \theta)^{-1 / 2}$ & $\left(2 / N_{\text {tot }}\right)^{1 / 2} /|\sin 2 \theta|$ & $2\left(2 / N_{t o t}\right)^{1 / 2}(3+\cos 4 \theta-2 \sin 4 \theta)^{-1 / 2}$ \\
\hline & dual & $2(2 / N \text { tot })^{1 / 2}(3+\cos 4 \theta+2 \sin 4 \theta)^{-1 / 2}$ & $\left(2 / N_{\text {tot }}\right)^{1 / 2} /|\sin 2 \theta|$ & $2(2 / N \text { tot })^{1 / 2}(3+\cos 4 \theta-2 \sin 4 \theta)^{-1 / 2}$ \\
\hline \multirow[t]{2}{*}{$w W^{\prime}$} & single & $4 / N_{\text {tot }}^{1 / 2}\left(\frac{3+\cos 4 \theta+2 \cos \zeta \sin 4 \theta}{15+12 \cos 4 \theta+5 \cos 8 \theta}\right)^{1 / 2}$ & $\left(2 / N_{t o t}\right)^{1 / 2} /|\sin 2 \theta|$ & $4 / N_{\text {tot }}^{1 / 2}\left(\frac{3+\cos 4 \theta-2 \cos \zeta \sin 4 \theta}{15+12 \cos 4 \theta+5 \cos 8 \theta}\right)^{1 / 2}$ \\
\hline & dual & $4 / N_{\text {tot }}^{1 / 2}\left(\frac{3+\cos 4 \theta+2 \cos \zeta \sin 4 \theta}{15+12 \cos 4 \theta+5 \cos 8 \theta}\right)^{1 / 2}$ & $\left(2 / N_{t o t}\right)^{1 / 2} /|\sin 2 \theta|$ & $4 / N_{\text {tot }}^{1 / 2}\left(\frac{3+\cos 4 \theta-2 \cos \zeta \sin 4 \theta}{15+12 \cos 4 \theta+5 \cos 8 \theta}\right)^{1 / 2}$ \\
\hline \multirow[t]{2}{*}{$w w^{\prime} W W^{\prime}$} & single & $2(2 / N \text { tot })^{1 / 2}(3+\cos 4 \theta+2 \sin 4 \theta)^{-1 / 2}$ & $\left(2 / N_{t o t}\right)^{1 / 2} /|\sin 2 \theta|$ & $2(2 / N \text { tot })^{1 / 2}(3+\cos 4 \theta-2 \sin 4 \theta)^{-1 / 2}$ \\
\hline & dual & $2(2 / N \text { tot })^{1 / 2}(3+\cos 4 \theta+2 \sin 4 \theta)^{-1 / 2}$ & $\left(2 / N_{t o t}\right)^{1 / 2} /|\sin 2 \theta|$ & $2(2 / N \text { tot })^{1 / 2}(3+\cos 4 \theta-2 \sin 4 \theta)^{-1 / 2}$ \\
\hline
\end{tabular}

Table 3. Error estimates for unnormalized Stokes parameters for different wedge configurations

\begin{tabular}{|c|c|c|c|c|c|}
\hline Weges & Beam & $\sigma(I)$ & $\sigma(Q)$ & $\sigma(U)$ & $\sigma(V)$ \\
\hline$q w$ & $\begin{array}{l}\text { single } \\
\text { dual }\end{array}$ & $2 N_{t o t}^{1 / 2} / n x$ & $2\left(2 N_{t o t}\right)^{1 / 2} / n x$ & $2\left(2 N_{t o t}\right)^{1 / 2} / n x$ & \\
\hline$q w w^{\prime}$ & $\begin{array}{l}\text { single } \\
\text { dual }\end{array}$ & $2 N_{t o t}^{1 / 2} / n x$ & $2\left(2 N_{t o t}\right)^{1 / 2} / n x$ & $2\left(2 N_{t o t}\right)^{1 / 2} / n x$ & \\
\hline$w W$ & $\begin{array}{l}\text { single } \\
\text { dual }\end{array}$ & $2 N_{t o t}^{1 / 2} / n x$ & $4\left(2 N_{t o t} /(3+\cos 4 \theta+2 \sin 4 \theta)\right)^{1 / 2} / n x$ & $2\left(2 N_{t o t}\right)^{1 / 2} /(n x|\sin 2 \theta|)$ & $4\left(2 N_{t o t} /(3+\cos 4 \theta-2 \sin 4 \theta)\right)^{1 / 2} / n x$ \\
\hline$w W^{\prime}$ & $\begin{array}{l}\text { single } \\
\text { dual }\end{array}$ & $2 N_{t o t}^{1 / 2} / n x$ & $\frac{8}{n x}\left(N_{t o t} \frac{3+\cos 4 \theta+2 \cos \zeta \sin 4 \theta}{15+12 \cos 4 \theta+5 \cos 8 \theta}\right)^{1 / 2}$ & $2\left(2 N_{t o t}\right)^{1 / 2} /(n x|\sin 2 \theta|)$ & $\frac{8}{n x}\left(N_{t o t} \frac{3+\cos 4 \theta-2 \cos \zeta \sin 4 \theta}{15+12 \cos 4 \theta+5 \cos 8 \theta}\right)^{1 / 2}$ \\
\hline$w w^{\prime} W W^{\prime}$ & $\begin{array}{l}\text { single } \\
\text { dual }\end{array}$ & $2 N_{t o t}^{1 / 2} / n x$ & $4\left(2 N_{t o t} /(3+\cos 4 \theta+2 \sin 4 \theta)\right)^{1 / 2} / n x$ & $2\left(2 N_{t o t}\right)^{1 / 2} /(n x|\sin 2 \theta|)$ & $4\left(2 N_{t o t} /(3+\cos 4 \theta-2 \sin 4 \theta)\right)^{1 / 2} / n x$ \\
\hline
\end{tabular}




\section{References}

1. F. Snik and C. Keller, "Astronomical Polarimetry" in Planets, Stars and Stellar Systems (Springer, 2012).

2. D. Goldstein, Polarized Light, 3rd Edition (CRC Press, 2011).

3. W. B. Sparks, J. H. Hough, T. A. Germer, F. Chen, S. DasSarma, P. DasSarma, F. Robb, N. Manset, L. Kolokolova, I. Reid, F. Macchetto, and W. Martin, "Detection of circular polarization in light scattered from photosynthetic microbes." Proc. Nat. Acad. Sci. 106, 7816-7821 (2009).

4. W. B. Sparks, J. H. Hough, L. Kolokolova, T. A. Germer, F. Chen, S. DasSarma, P. DasSarma, F. T. Robb, N. Manset, I. N. Reid, F. D. Macchetto, and W. Martin, "Circular polarization in scattered light as a possible biomarker," J. Quant. Spect. Rad. Trans. 110, 1771-1779 (2009).

5. F. Snik, T. Karalidi, and C. Keller, "Spectral modulation for full linear polarimetry." Applied Optics 48, 1337-1346 (2009).

6. G. van Harten, F. Snik, J. H. H. Rietjens, J. M. Smit, J. de Boer, R. Diamantopoulou, O. P. Hasekamp, D. M. Stam, C. U. Keller, E. C. Laan, A. L. Verlaan, W. A. Vliegenthart, R. Ter Horst, R. Navarro, K. Wielinga, S. Hannemann, S. G. Moon, and R. Voors, "Prototyping for the Spectropolarimeter for Planetary EXploration (SPEX): calibration and sky measurements," in "Society of Photo-Optical Instrumentation Engineers (SPIE) Conference Series", vol. 8160 of Society of Photo-Optical Instrumentation Engineers (SPIE) Conference Series (2011).

7. K. Oka and T. Kaneko, "Compact complete imaging polarimeter using birefringent wedge prisms." Optics Express 11, 1510-1519 (2003).

8. K. Serkowski, "A polarimetric method of measuring radial velocities." Pub. Astron. Soc. Pac. 84, 649-651 (1972).

9. K. Nordseick, "A simple polarimetric system for the lick observatory image-tube scanner." Pub. Astron. Soc. Pac. 86, 324-329-2249 (1974).

10. K. Oka and T. Kato, "Spectroscopic polarimetry with a channeled spectrum." Optics Letters 24, 1475-1477 (1999).

11. R. W. Oka and N. Saito, "Snapshot complete imaging polarimeter using savart plates." Infrared Detectors and Focal Plane Arrays VIII. Eds. Dereniak, E.L.; Sampson, R.E., Proc. SPIE 6295, 629508 (2006).

12. M. Mujat, E. Baleine, and A. Dogariu, "Interferometric imaging polarimeter." J. Opt. Soc. Am. 21, 2244-2249 (2004).

13. T. Wakayama, Y. Otani, and N. Umeda, "One-shot birefringence dispersion measurement based on channeled spectrum technique." Optics Communications 281, 3668-3672 
(2008).

14. J. Howard, "Snapshot-imaging motional stark effect polarimetry." Plasma Phys. Control Fusion 50, 125003 (2008).

15. M. W. Kudenov, L. Pezzaniti, E. L. Dereniak, and G. R. Gerhart, "Prismatic imaging polarimeter calibration for the infrared spectral region," Optics Express 16, 13720 (2008).

16. F. Snik, J. H. H. Rietjens, G. van Harten, D. M. Stam, C. U. Keller, J. M. Smit, E. C. Laan, A. L. Verlaan, R. Ter Horst, R. Navarro, K. Wielinga, S. G. Moon, and R. Voors, "SPEX: the spectropolarimeter for planetary exploration," in "Society of Photo-Optical Instrumentation Engineers (SPIE) Conference Series," vol. 7731 of Society of PhotoOptical Instrumentation Engineers (SPIE) Conference Series (2010).

17. E. H. Geyer, K. Jockers, N. N. Kiselev, and G. P. Chernova, "A novel quadruple beam imaging polarimeter and its application to Comet Tanaka-Machholz 1992 X," apss 239, 259-274 (1996).

18. E. Oliva, "Wedged double Wollaston, a device for single shot polarimetric measurements," aaps 123, 589-592 (1997).

19. C. Pernechele, E. Giro, and D. Fantinel, "Device for optical linear polarization measurements with a single exposure," in "Society of Photo-Optical Instrumentation Engineers (SPIE) Conference Series", vol. 4843 of Society of Photo-Optical Instrumentation Engineers (SPIE) Conference Series, S. Fineschi, ed. (2003), pp. 156-163.

20. T. M. Brown, D. Charbonneau, R. L. Gilliland, R. W. Noyes, and A. Burrows, "Hubble Space Telescope Time-Series Photometry of the Transiting Planet of HD 209458," Astrophys. J. 552, 699-709 (2001).

21. J. M. Beckers, "Variations in the Birefringence of Quartz," Appl. Opt. 6, 1279 (1967).

22. B. Boulbry, J. C. Ramella-Roman, and T. A. Germer, "Improved method for calibrating a Stokes polarimeter," Appl. Opt. 46, 8533-8541 (2007).

23. P. Bevington, Data Reduction and Error Analysis for the Physical Sciences, 1st Edition (McGraw-Hill, 1968).

24. P. Bevington and D. Robinson, Data Reduction and Error Analysis for the Physical Sciences, 3rd Edition (McGraw-Hill, 2002). 


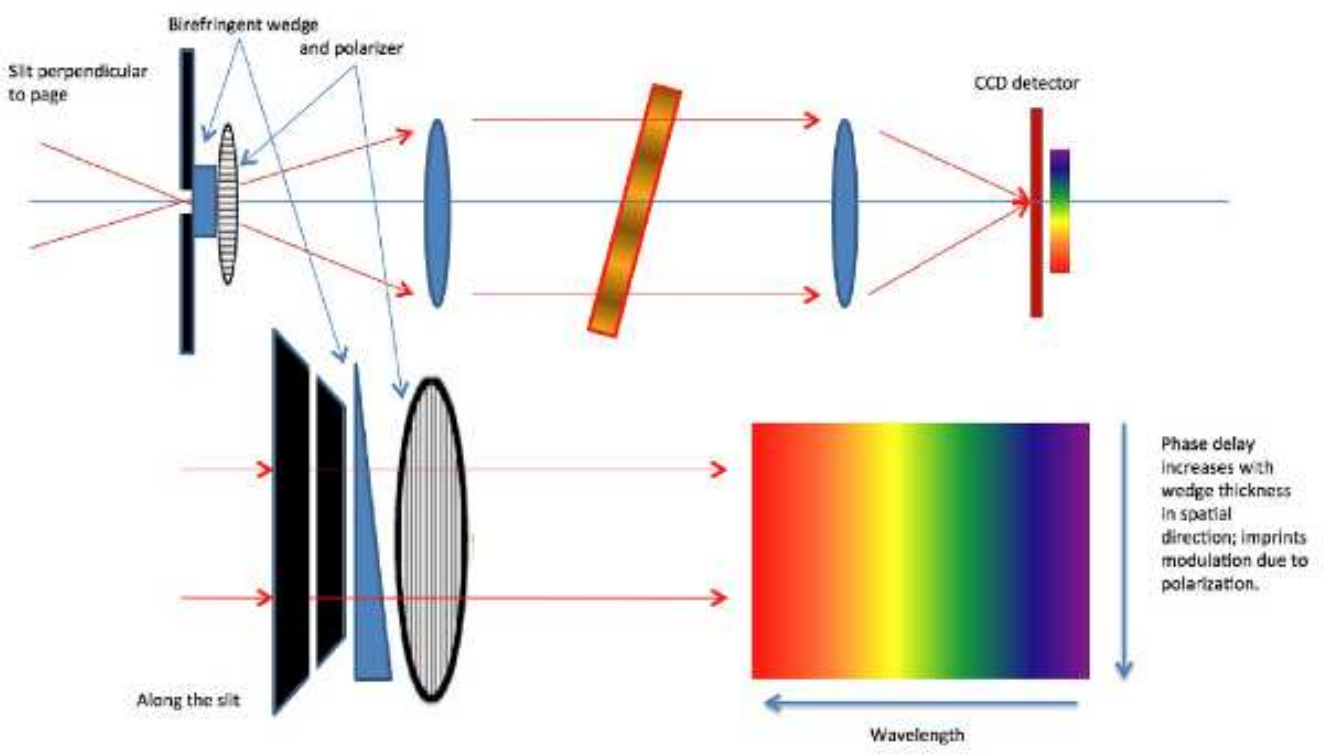

Fig. 1. Illustration of the optical bench layout. Light enters a spatially elongated slit, passes through a birefringent wedge or wedges and polarization analyzer, and then enters a conventional long-slit spectrograph. A quarter wave retarder (not shown) may be inserted before the wedge. 


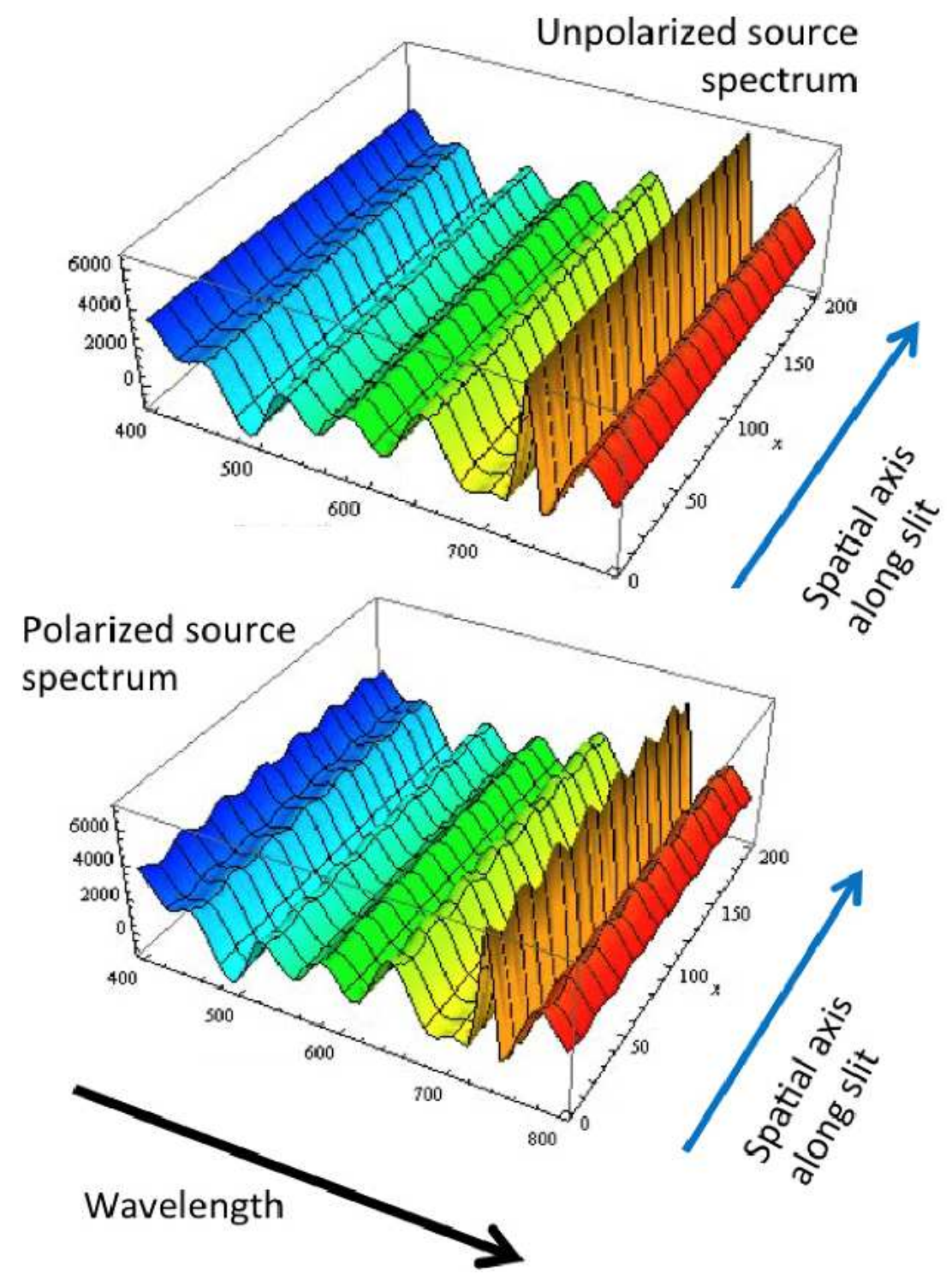

Fig. 2. Concept - the polarization optics imprint an amplitude modulation on the dimension orthogonal to the dispersion direction of the spectrograph. Typically, this direction corresponds to the spatial dimension along the slit. 

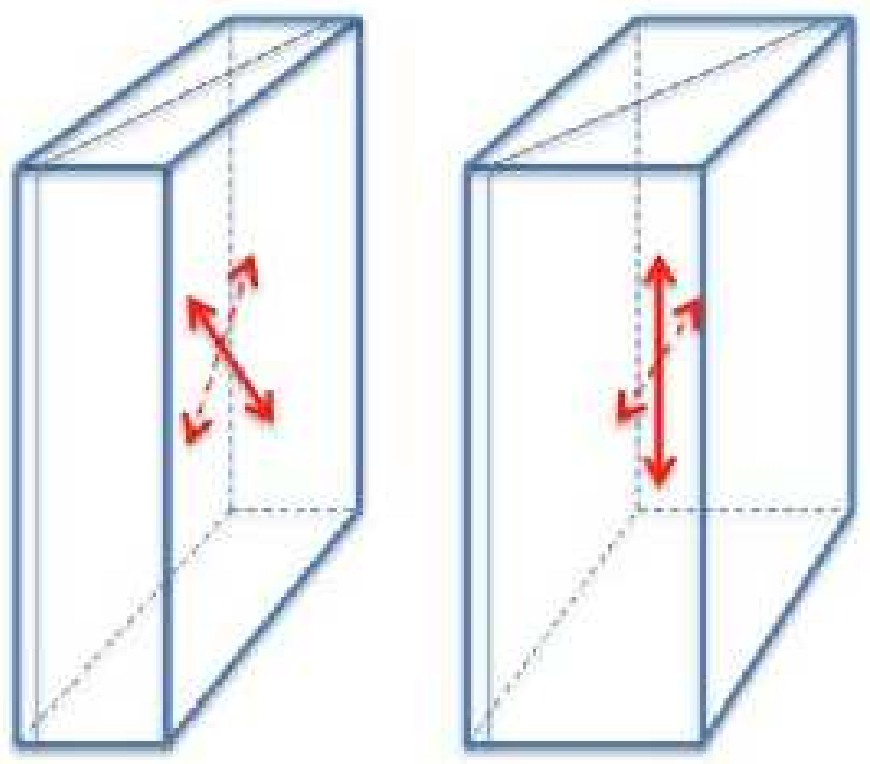

Fig. 3. Illustration of the compound birefringent wedges. A single compound optic may be used for linear polarimetry (left) and double (both) for full Stokes. The fast axes run at $\pm 45^{\circ}$ in the left optic, horizontal and vertical in the right optic, and the slit direction is horizontal or vertical. 


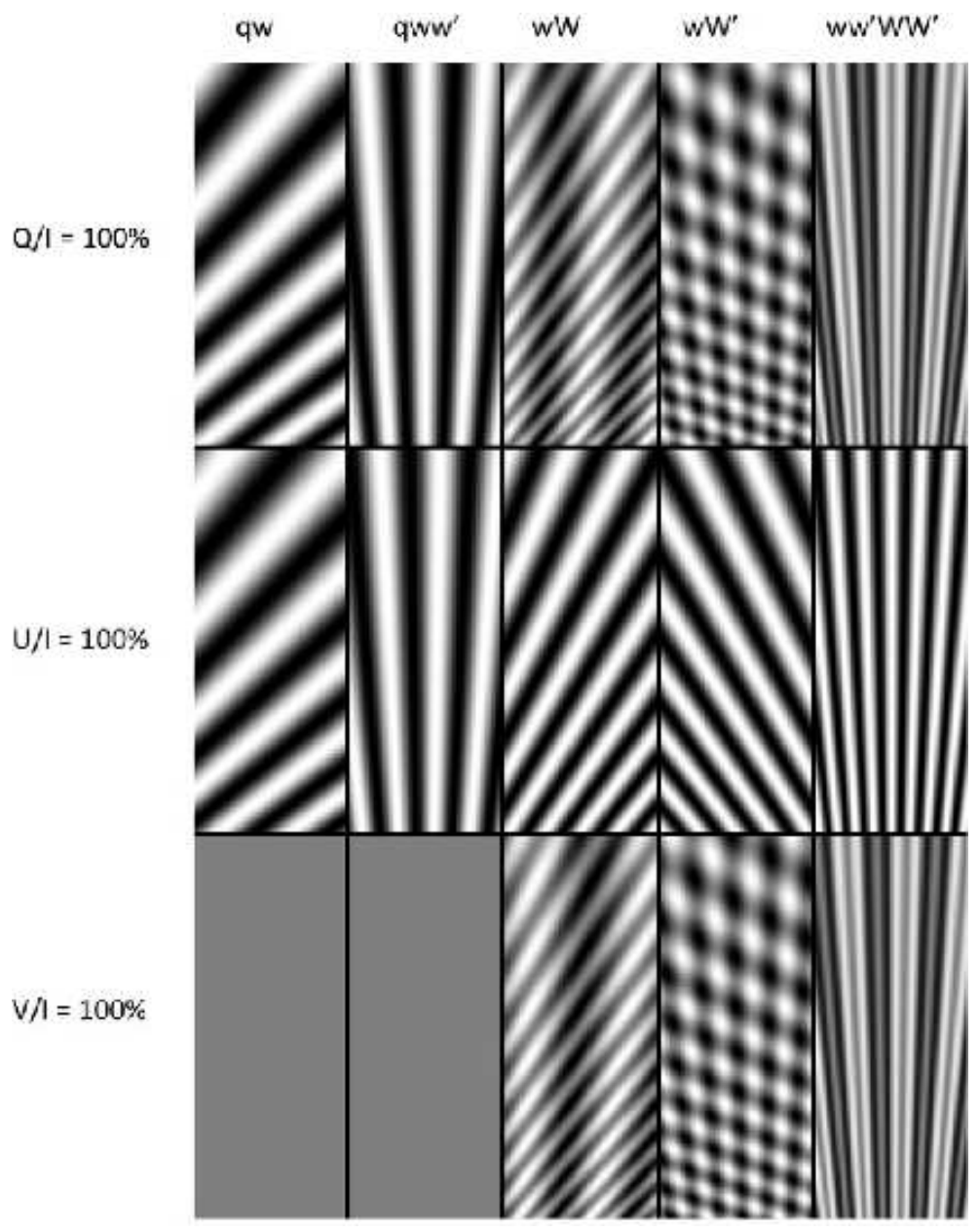

Fig. 4. Example theoretical data frames for each of the configurations discussed in the text when viewed with $100 \%$ polarized light. Each panel has $x$ running horizontally and wavelength vertically, increasing up. Parameters correspond to $2 \mathrm{~mm}$ in $x$ of a $3^{\circ}$ quartz wedge set, running from $450 \mathrm{~nm}$ to $750 \mathrm{~nm}$. Top row shows 100\% Stokes Q, middle row 100\% Stokes $U$ and bottom row $100 \%$ Stokes $V$. Left to right, in the notation of Tables 1-3, the configurations are $q w, q w w^{\prime}, w W, w W^{\prime}$, and $w w^{\prime} W W^{\prime}$. Note that if the quarter wave retarder were omitted in the first two columns, $U$ and $V$, which show no sensitivity with the quarter wave retarder, would be interchanged. For the first two columns, the fast axis is set at $0^{\circ}$ and for the remaining three at $45^{\circ}$ (see text). 


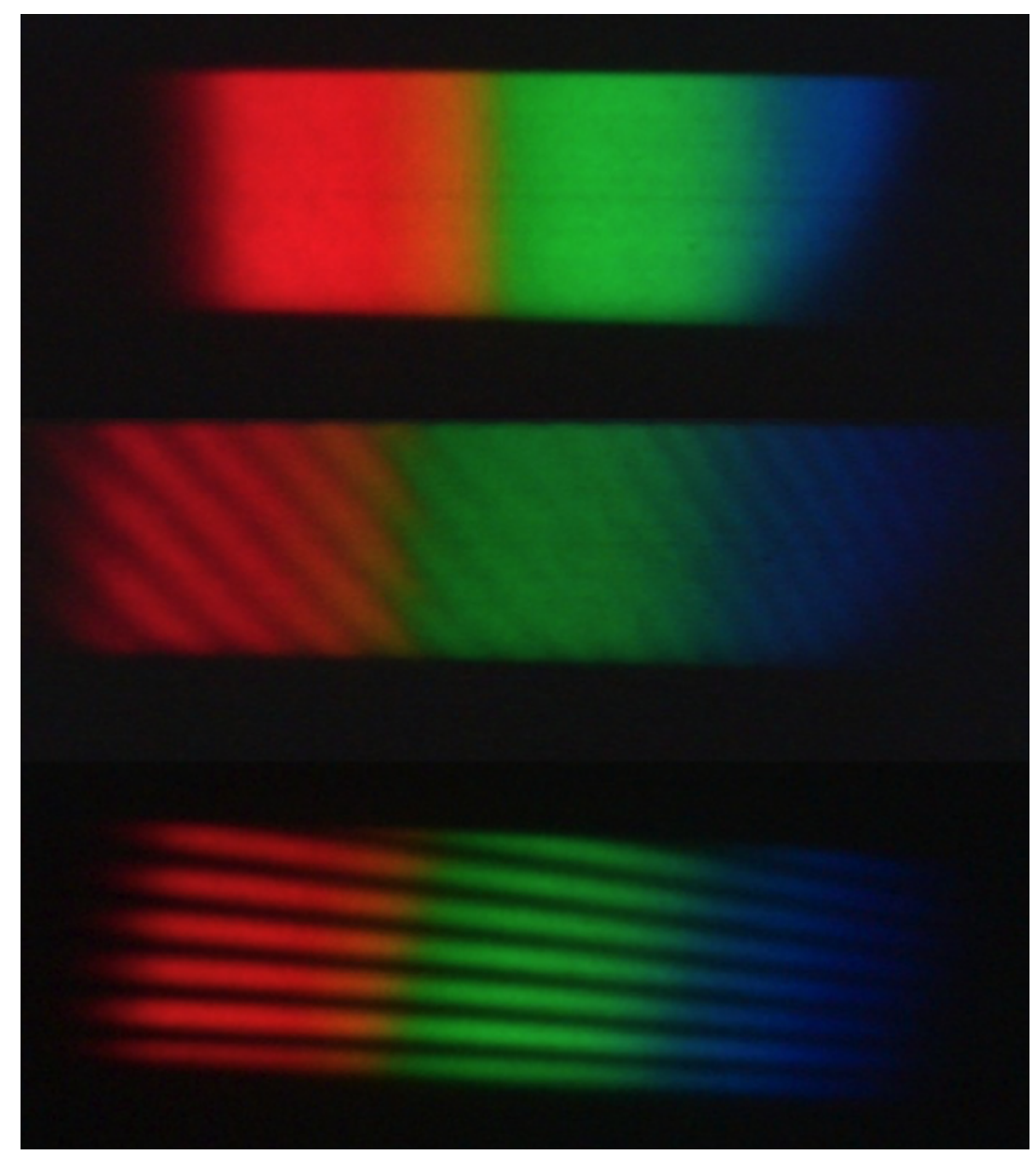

Fig. 5. Actual spectra obtained with a preliminary test optical bench: (upper) a spectrum obtained with unpolarized light, (center) a spectrum with one quartz birefringent wedge and $100 \%$ linearly polarized light, and (lower) a spectrum obtained with two quartz birefringent wedges reversed as in the manner of the compound optics with $100 \%$ linearly polarized light. 


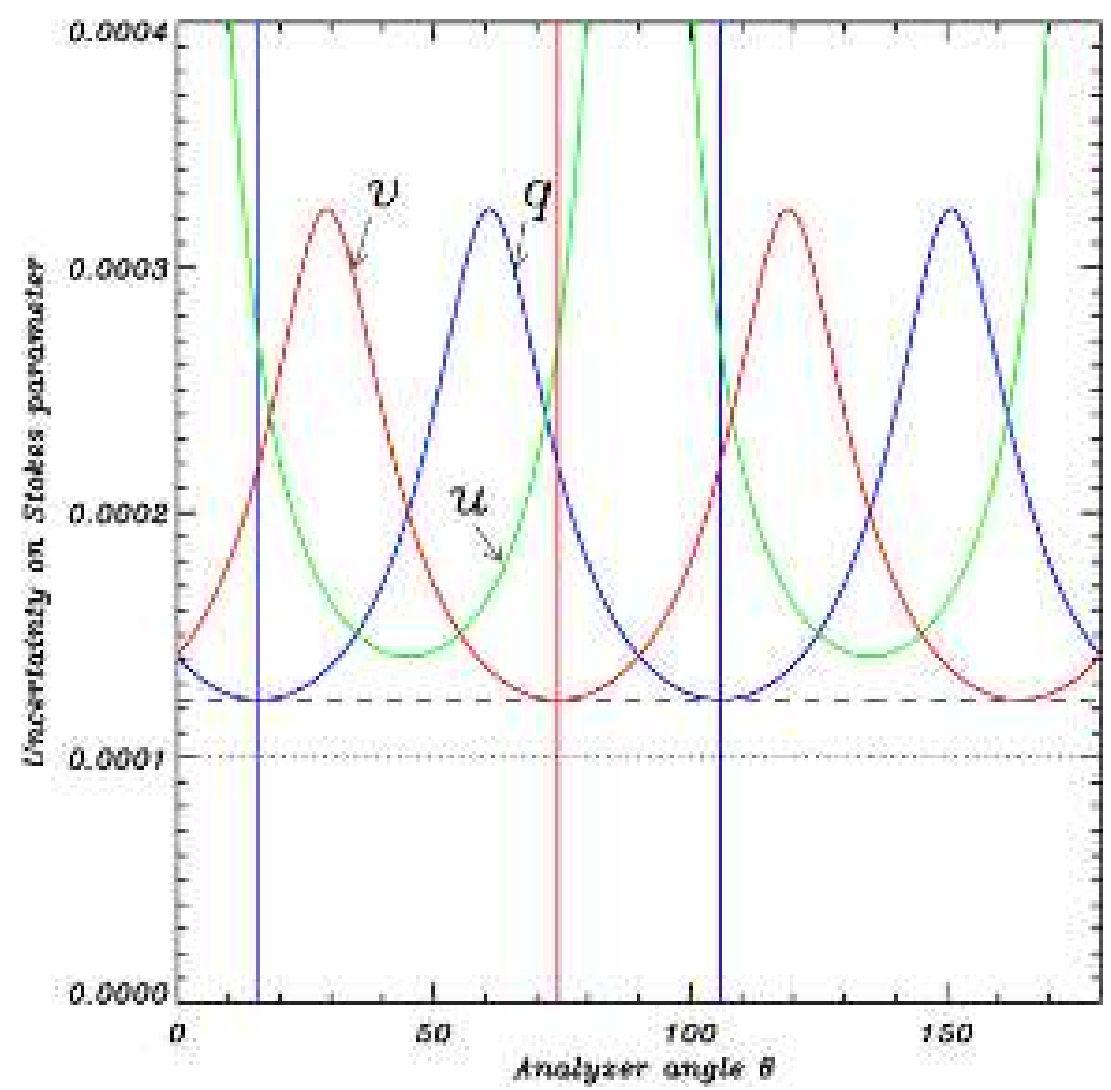

Fig. 6. The uncertainties on normalized Stokes parameters for double wedge pair configuration, Sec. 4.B.3. Blue is Stokes $q$, green is $u$, and red is $v$. The vertical lines indicate the positions of the minima for $q$ and $v$, the horizontal lines indicates $1 / \sqrt{N_{t o t}}$ (dotted), and the analytically-determined minimum $1.24 \times$ higher (dashed). 


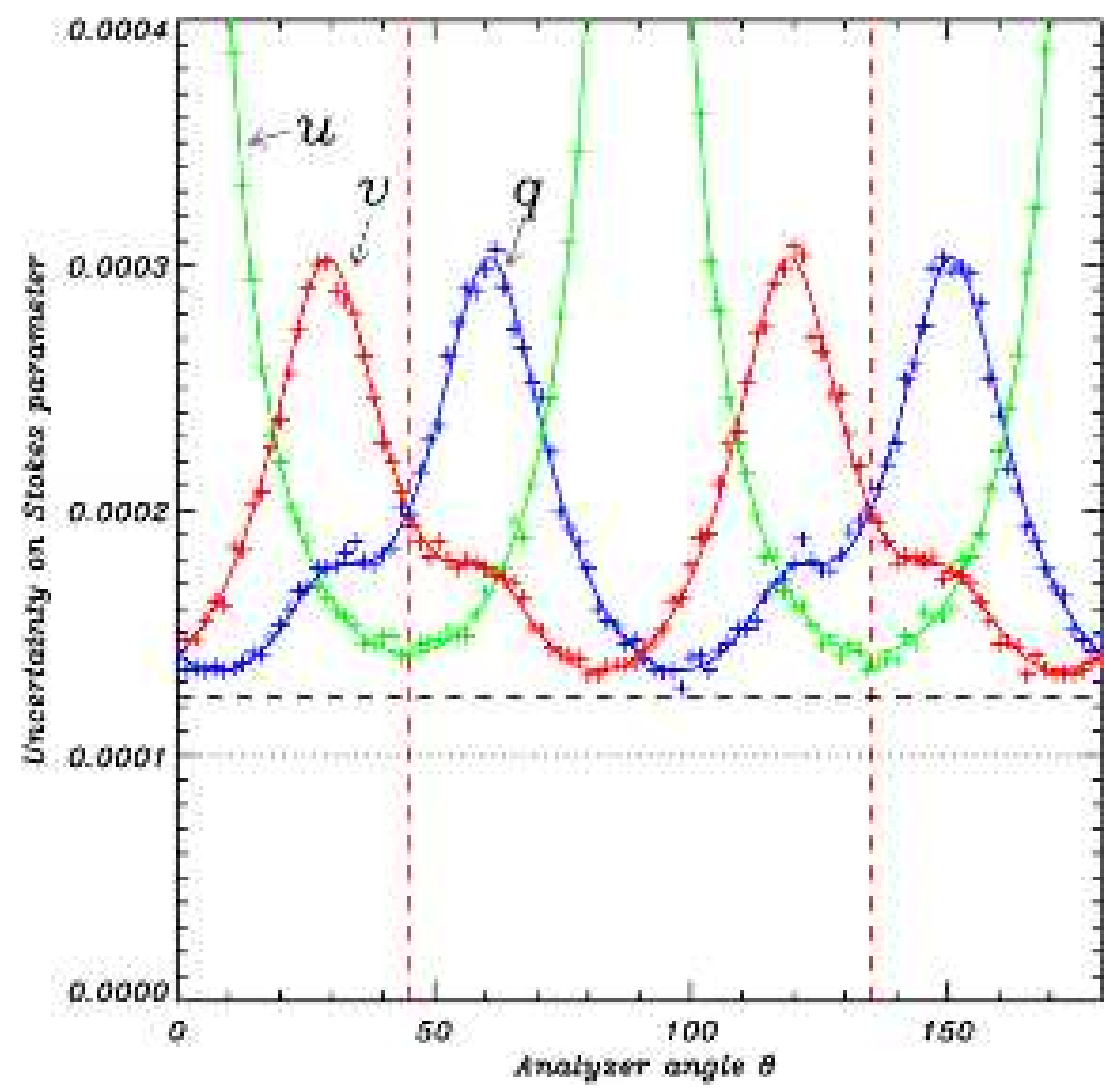

Fig. 7. The uncertainties on normalized Stokes parameters for double wedge pair configuration, Sec. 4.B.3 with miscentering of order $1 / 32 \times$ the spatial distance of one wavelength of retardance. Blue is Stokes $q$, green is $u$, and red is $v$. The vertical lines indicate the positions of the analyzer angles that have no formal covariance, which is independent of the miscentering. Smooth lines through the simulated data are the analytic solutions ignoring covariance terms, while the plus signs are the results of Monte-Carlo simulations. The horizontal lines are as in the previous figure. 

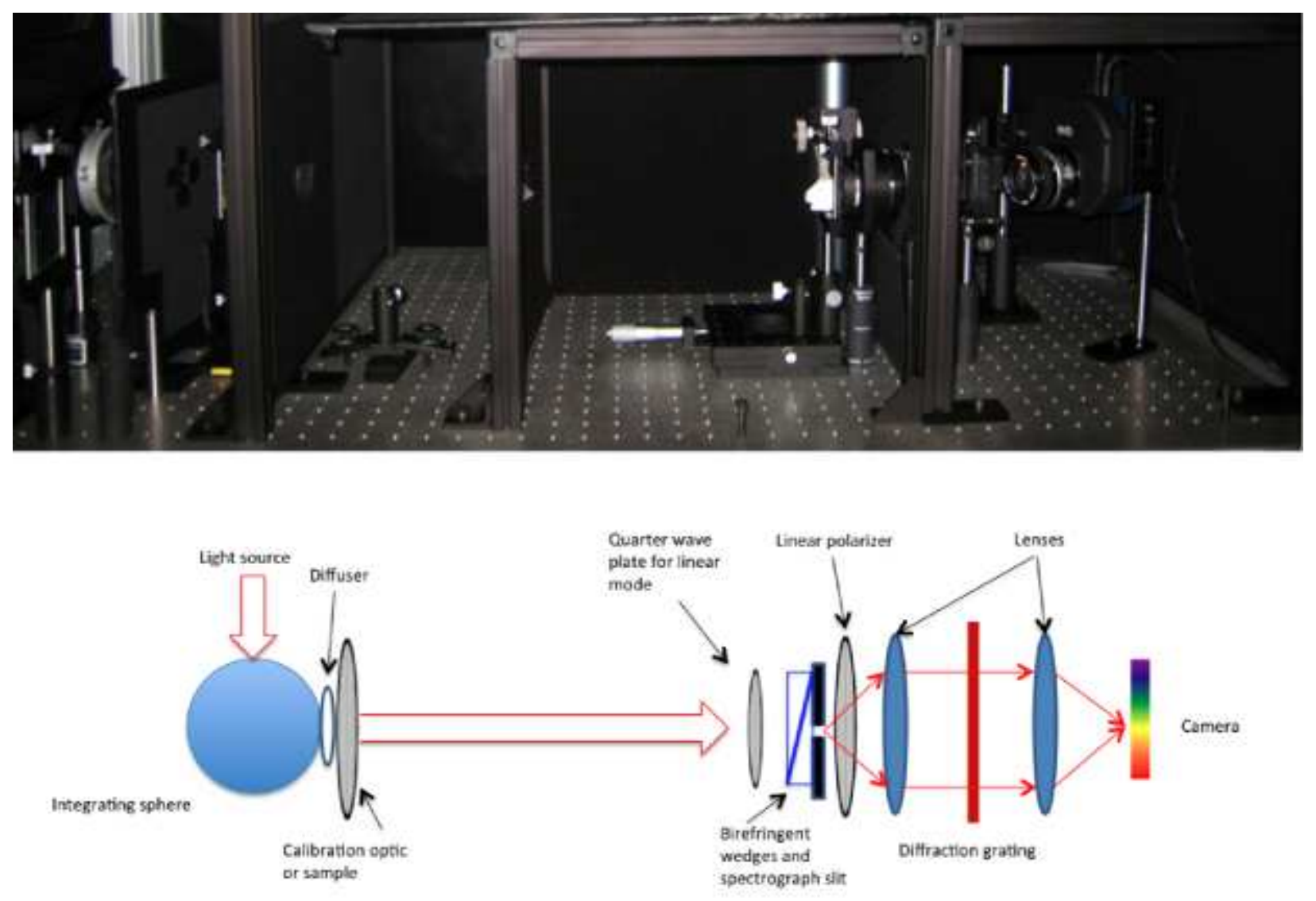

Fig. 8. Laboratory optical bench layout as implemented. Light enters an integrating sphere, and illuminates a diffusing screen on exit. It then passes through calibration or sample components, before entering the long slit spectrograph with its associated polarization components as described in the text. The upper panel shows the actual optical bench with baffles and boxes removed for visibility. The integrating sphere is to the left, and the camera to the right. 


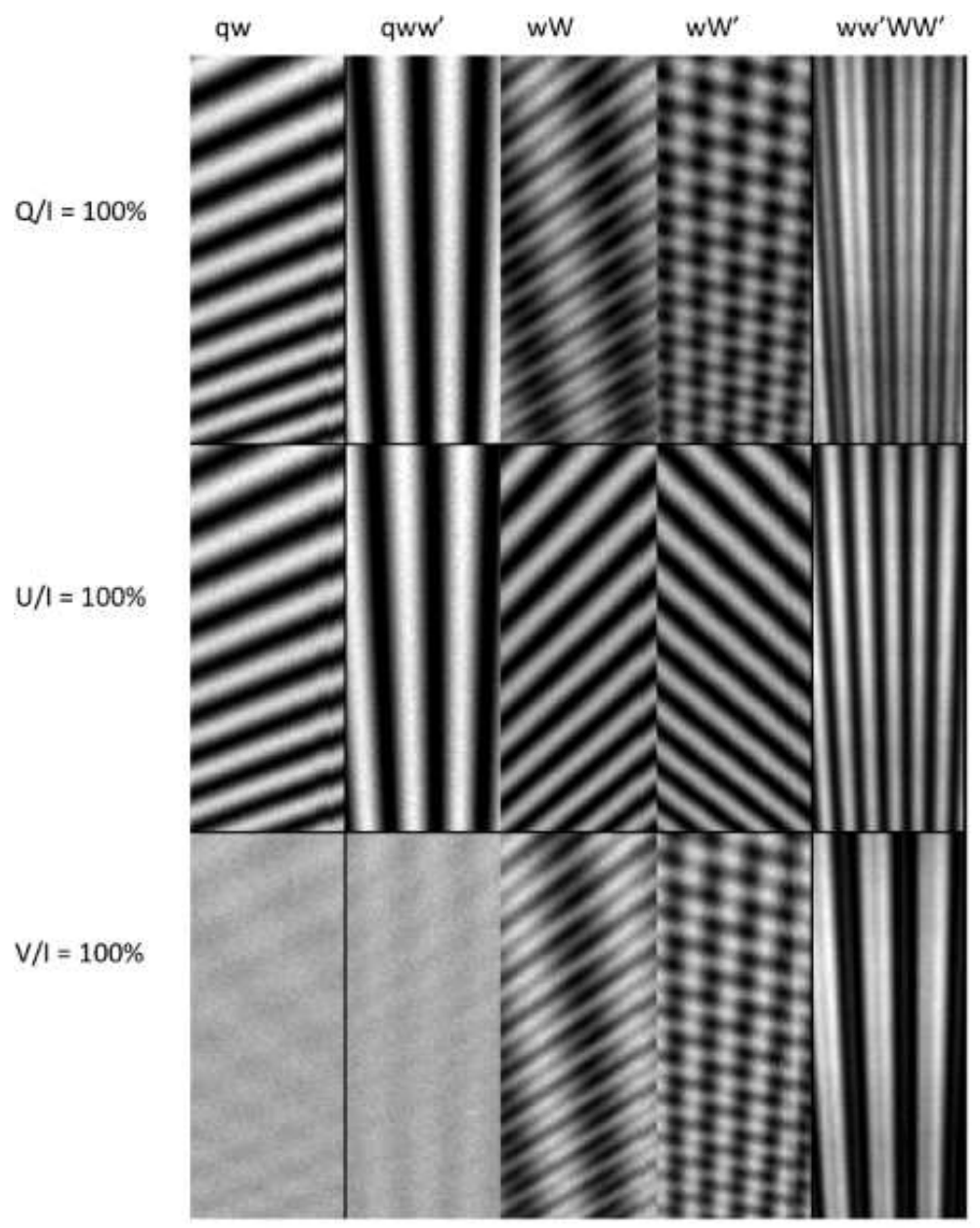

Fig. 9. Example data frames for each of the configurations discussed in the text when viewed with $\approx 100 \%$ polarized light, obtained in the laboratory. The rows and columns correspond to those shown in Fig. 4. 


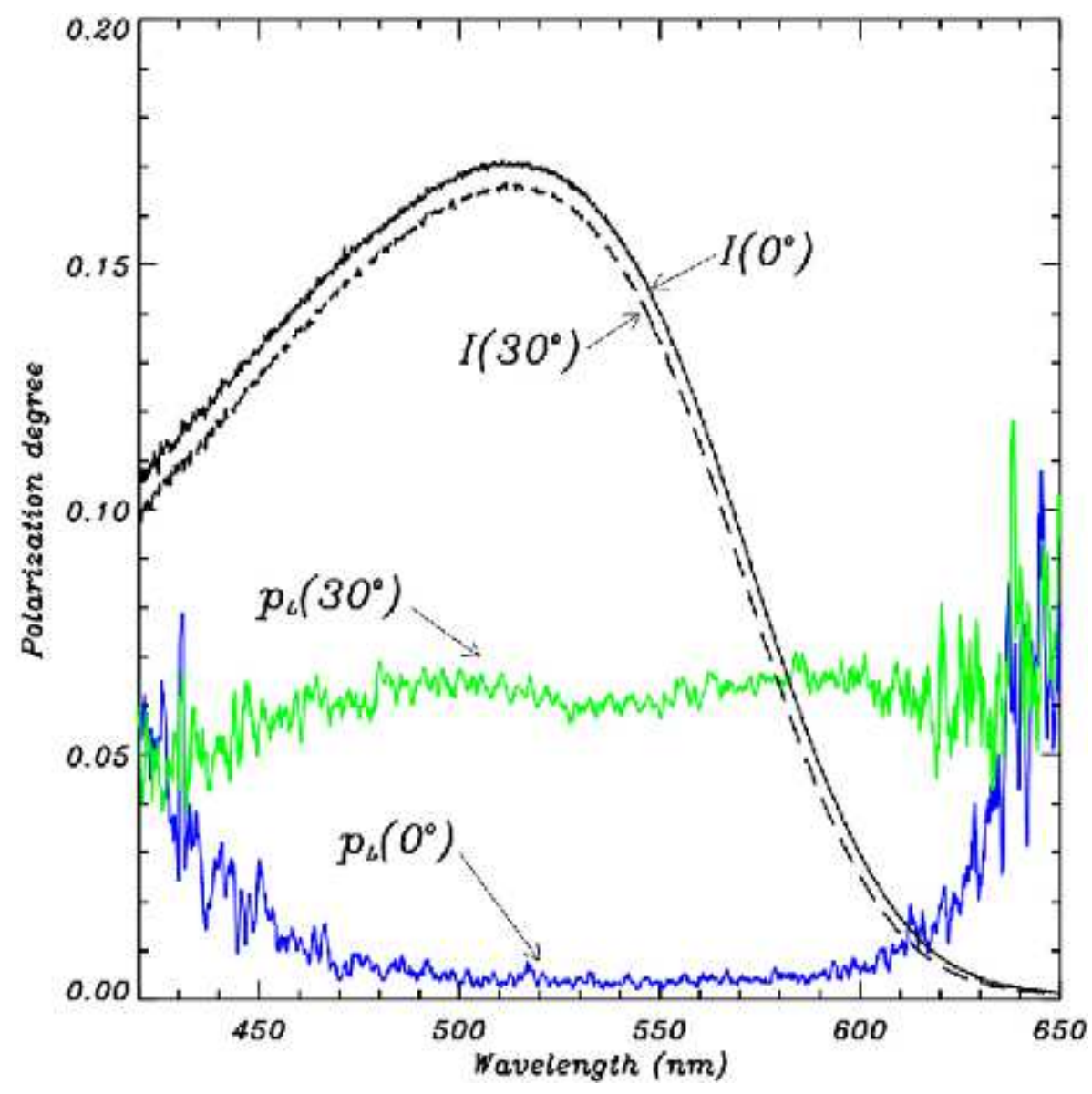

Fig. 10. Retrieved polarization curves for a BG18 colored glass filter presented orthogonal to the beam, blue, and at an angle tilted by $\approx 30^{\circ}$ to orthogonal, green, observed using the $w W^{\prime}$ configuration. At right angles, we expect no polarization, and inclined at $30^{\circ}$, approximately $6.3 \%$, consistent with the least squares retrieval. The black curves show arbitrarily normalized throughputs for the two configurations (solid, orthogonal and dotted, inclined) derived from the data, serving to illustrate that we also obtain full Stokes $I$ spectroscopy using these methods. 


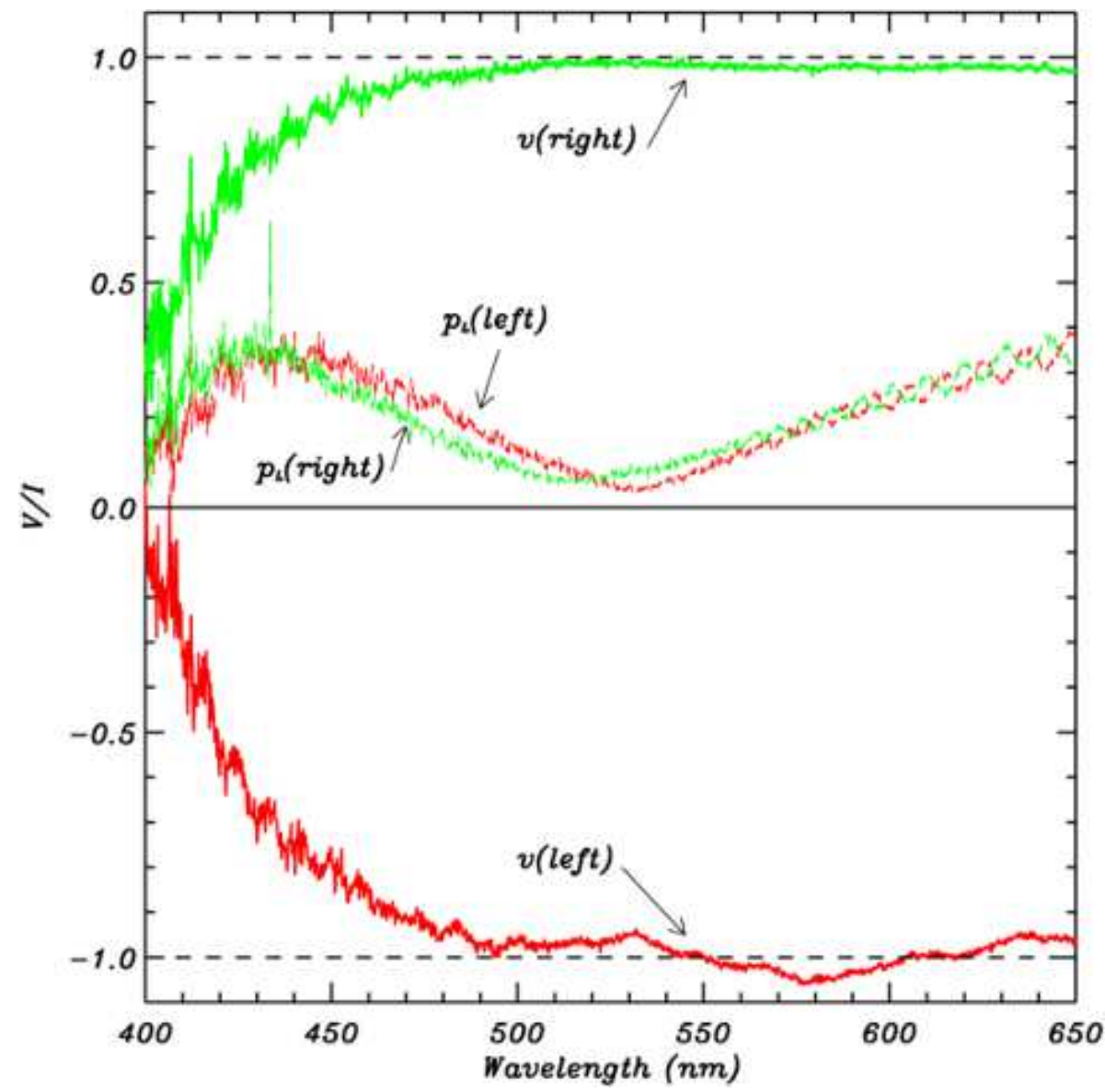

Fig. 11. Retrieved circular polarization for a pair of polarizing cinema 3D glasses, expected to exhibit 100\% Stokes $V$ left and right circularly polarized light for the left and right eyes, measured using the $w w^{\prime} W W^{\prime}$ configuration. The retrieval is consistent with expectations. For completeness, and to illustrate that we obtain full Stokes polarimetry from a single data frame, the dashed lines show the retrieved degree of linear polarization. 